\title{
Foundations of self-consistent particle-rotor models and of self-consistent cranking models
}

\author{
Abraham Klein* \\ Department of Physics, University of Pennsylvania, Philadelphia, PA 19104-6396, USA
}

\begin{abstract}
The Kerman-Klein formulation of the equations of motion for a nuclear shell model and its associated variational principle are reviewed briefly. It is then applied to the derivation of the self-consistent particle-rotor model and of the self-consistent cranking model, for both axially symmetric and triaxial nuclei. Two derivations of the particle-rotor model are given. One of these is of a form that lends itself to an expansion of the result in powers of the ratio of single-particle angular momentum to collective angular momentum, that is essential to reach the cranking limit. The derivation also requires a distinct, angular-momentum violating, step. The structure of the result implies the possibility of tilted-axis cranking for the axial case and full three-dimensional cranking for the triaxial one. The final equations remain number conserving. In an appendix, the Kerman-Klein method is developed in more detail, and the outlines of several algorithms for obtaining solutions of the associated non-linear formalism are suggested.
\end{abstract}

*Email: aklein@nucth.physics.upenn.edu 


\section{INTRODUCTION}

The aims of this paper are to study anew the foundations of the particle-rotor model [1:2] and of the cranking model [2]. The particle-rotor model (PRM) was introduced as an angular momentum-conserving phenomenological description of odd deformed nuclei. Because of its relative ease of application and, on the whole, quite remarkable success, it has been applied even up to the present (for instance [3]), with various alterations of detail, to a myriad of applications, over a lifetime of more than four and a half decades. Among the extensions, we mention in particular that to the description of triaxial nuclei [ [ 6 , the original model having been formulated for axially symmetric nuclei.

In one of the textbooks [2], p. 109, we find, after a glowing appraisal of the success of the model, the following statement: "However, until now a clear-cut microscopic derivation has been missing." In fact, a microscopic derivation had been given earlier [7], based on the Kerman-Klein (KK) method [8 [12]. The microscopic foundation of the axially symmetric PRM was studied more recently in [13], starting from a semi-microscopic version of the KK approach, and compared in accuracy, for several examples of well-deformed nuclei, both with its more accurate progenitor and with the inherently less accurate cranking approximation.

The cranking model was originally introduced into nuclear physics [14], within the framework of a prescribed single-particle model, to deal with the enigma presented by the first values encountered for the moments of inertia of deformed nuclei. An extended version [15], the one considered in most applications until recent years, was based on the self-consistent mean-field theory of a deformed rotating object. This early work was designed primarily to provide formulas for the moment of inertia.

The full range of applicability of the self-consistent cranking model, as well as its limitations, was realized in the so-called cranked shell model (CSM) [16], that has been widely applied to the analysis of band-crossing and other high-spin phenomena. (For a current list of references, especially reviews, see [17].) The formulations under discussion, which apply to axially symmetric nuclei, assume that collective rotation occurs about a principal axis perpendicular to the symmetry axis. Such a formulation is referred to currently as principalaxis cranking (PAC) as opposed to a recent generalization, called tilted-axis cranking (TAC) [17 21,?]. In the latter, even in the axial case, the system may rotate about an axis in a principal plane of the assumed (quadrupole) intrinsic shape, and for the triaxial case about an arbitrary (dynamically determined) direction with respect to the principal axes.

A second aim of the present paper is to establish the relationship of the cranking models, including the recent generalized versions, to a microscopic theory. The previous literature on this subject is modest in extent. The standard references are [22,23], the major results of which are reproduced and discussed in [2]. Briefly, starting from a formulation of the microscopic theory by means of generator coordinates, the energy is evaluated approximately as a power series in the angular momentum by a method due to Kamlah [24], valid for large deformations. When the variational method is applied to the lowest non-trivial approximation of this procedure, it can be shown that the cranking theory is a solution of the resulting equations. This is summarized by stating that cranking is a solution, involving a semi-classical approximation, of the method of variation after projection as opposed to the exact procedure of variation before projection.

To our knowledge, the only other studies of this subject are those based on the KK 
method, a brief treatment of the case of rotation in a plane 25] that predates the abovecited work and two studies that postdated them, one again on the problem of rotation in a plane [26] and the second a restricted study of the triaxial case [27]. (Some discussion of the cranking limit, also based on a variant of the KK method, can be found in [29].) Up to now we have never presented a full account of the three-dimensional treatment either for axial or for triaxial nuclei. Since our methods contain features distinct from those found in the standard literature [2], and in view of the renewed interest in generalized cranking models 1721 , the publication of a detailed account is perhaps justified, even at this late date.

The foundations of the study are presented in Sec. II. We utilize a shell model Hamiltonian, widely employed for medium and heavy nuclei, with two-particle interactions in which the latter are separated into two parts clearly distinguished as multipole and pairing forces, respectively. The advantage of such a model is that the (c-number) equations of motion that can be derived from it by the KK method are completely rigorous. It is a simplifying feature for the further study to recognize that these equations can be derived from a variational principle that we called the trace variational principle, suggested in our earliest paper [8] and developed more fully in [28] and in [29]. This variational principle has several noteworthy features: (i) It is formulated for the many-body problem in the language of second quantization. (ii) The quantities varied are not wave functions, but rather a suitably chosen set of matrix elements, in our case coefficients of fractional parentage (to be discussed at the appropriate point of Sec. II). (iii) Rather than involving the Rayleigh-Ritz principle for one state at a time, the functional to be varied is the trace of energy expectation values over a prescribed space of states.

[It turns out that not all aspects of our formulation are novel. Thus an incomplete version of the trace variational principle is to be found in one of the initial series of papers on matrix mechanics [30], in which the variational parameters are matrix elements of the coordinates and momenta. This application to particle quantum mechanics was discovered and developed independently by us in several accounts of which the most recent is [31]. A version of the trace variational principle can, furthermore, be found is in a classic text in mathematical physics 32]. Here the formulation is close to standard Rayleigh-Ritz, in that the quantities varied are wave function. This formulation has found its way into the theory of density functionals [33] and even been generalized to include the case where the trace is replaced by a different weighted diagonal sum [34]. Most recently the trace variational principle for fields has appeared in a quaternion generalization of quantum mechanics 35 .]

The theory is elaborated in Sec. II only as far as is required for the remaining body of the text. Further development is presented in Appendix A, with an eye to formulating algorithms that can eventually be applied to the study of the self-consistent problem posed by the formulation in Sec. II. We turn to applications in Sec. III, where we derive the selfconsistent PRM from the variational principle associated with the KK equations. (With one possible exception [36], we are unaware of any recent work, other than our own, that has examined the foundations of the PRM.)

The formalism presented in Sec. III does not lend itself naturally to a derivation of the self-consistent cranking theory, which should be a limit of the self-consistent PRM. In Sec. IV we describe an alternative derivation of the PRM, following ideas first advanced briefly in [25], that does lead directly to the cranking limit. The considerations of Secs. III and IV apply to axially symmetric nuclei. Both treatments are extended to the case of triaxial nuclei in Sec. V. Further discussion of results and conclusions are given in Sec. VI. 


\section{EQUATIONS OF MOTION AND VARIATIONAL PRINCIPLE}

We choose a shell-model Hamiltonian in the form

$$
H=h_{a} a_{\alpha}^{\dagger} a_{\alpha}+\frac{1}{2} F_{\alpha \gamma \delta \beta} a_{\alpha}^{\dagger} a_{\gamma} a_{\beta}^{\dagger} a_{\delta}+\frac{1}{2} G_{\alpha \gamma \beta \delta} a_{\alpha}^{\dagger} a_{\gamma}^{\dagger} a_{\delta} a_{\beta}
$$

In this standard model, the $a_{\alpha}, a_{\alpha}^{\dagger}$ are the destruction, creation operators for fermions in the shell-model mode $\alpha=(n l j m \tau)$ ( $\tau$ distinguishing neutrons from protons); $F_{\alpha \gamma \delta \beta}$ describes multipole forces and $G_{\alpha \gamma \delta \beta}$ pairing forces. In this version, all multipolarities allowed by angular momentum conservation are included, though in practice we limit ourselves to the lowest few multipoles of each type. We shall also consistently use the summation convention, except when we wish to highlight some set of indices. With the help of the definitions

$$
\begin{aligned}
F_{\alpha \gamma \delta \beta} & =s_{\gamma}\left(j_{a} m_{a} j_{c}-m_{c} \mid L M_{L}\right) s_{\beta}\left(j_{d} m_{d} j_{b}-m_{b} \mid L M_{L}\right) F_{a c d b}(L), \\
G_{\alpha \gamma \delta \beta} & =\left(j_{a} m_{a} j_{c} m_{c} \mid L M_{L}\right)\left(j_{d} m_{d} j_{b} m_{b} \mid L M_{L}\right) G_{a c d b}(L), \\
s_{\gamma} & =(-1)^{j_{c}-m_{c}}=\sqrt{2 j_{c}+1}\left(j_{c} m_{c} j_{c}-m_{c} \mid 00\right),
\end{aligned}
$$

where $\left(j m j^{\prime} m^{\prime} \mid L M\right)$ is a Clebsch-Gordon (CG) coefficient, the operator equations of motion can be obtained in the form

$$
\begin{aligned}
{\left[a_{\alpha}, H\right] } & =h_{a}^{\prime} a_{\alpha}+F_{\alpha \alpha^{\prime} \beta^{\prime} \beta} a_{\alpha^{\prime}} a_{\beta}^{\dagger} a_{\beta^{\prime}}+G_{\alpha \alpha^{\prime} \beta \beta^{\prime}} a_{\alpha^{\prime}}^{\dagger} a_{\beta^{\prime}} a_{\beta}, \\
h_{a}^{\prime} & =h_{a}-\frac{1}{2} F_{a b a b} \frac{2 L+1}{2 j_{a}+1}, \\
{\left[a_{\bar{\alpha}}^{\dagger}, H\right] } & =-h_{a}^{\prime \prime} a_{\bar{\alpha}}^{\dagger}-F_{\beta \beta^{\prime} \alpha^{\prime} \bar{\alpha}} a_{\beta^{\prime}}^{\dagger} a_{\beta} a_{\alpha^{\prime}}^{\dagger}-G_{\beta \bar{\beta}^{\prime} \alpha^{\prime} \bar{\alpha}} a_{\alpha^{\prime}} a_{\beta}^{\dagger} a_{\bar{\beta}^{\prime}}^{\dagger}, \\
h_{a}^{\prime \prime} & =h_{a}^{\prime}+2 \frac{2 L+1}{2 j_{a}+1} G_{a b a b}(L) .
\end{aligned}
$$

Here, for example, $\bar{\alpha}=\left(j_{a},-m_{a}\right)$.

To develop a dynamical scheme, we turn to the problem of obtaining equations for the matrix elements of Eqs. (2.5) and (2.7). We designate a state of interest of an odd nucleus as $|J M \nu\rangle$, where $J$ is the total angular momentum, $M$ is its $z$ component, and $\nu$ are the remaining quantum numbers necessary for unique specification of the state. Neighboring even nuclei are specified, correspondingly, as $|\overline{I M n}\rangle$, referring to a heavier neighbor, and $|\underline{I M n}\rangle$, referring to a lighter neighbor. Below we shall then derive equations for the matrix elements, referred to as CFP (coefficients of fractional parentage)

$$
\begin{aligned}
& \left\langle J M \nu\left|a_{\alpha}\right| \overline{I M_{I} n}\right\rangle=V_{J M \nu}\left(\alpha I M_{I} n\right), \\
& \left\langle J M \nu\left|a_{\bar{\alpha}}^{\dagger}\right| \underline{I M_{I} n}\right\rangle=U_{J M \nu}\left(\alpha I M_{I} n\right) .
\end{aligned}
$$

We shall require the full notation when we turn to applications in the next section. For the formal developments of this section, we utilize a compressed notation, with

$$
J M \nu \rightarrow i, \quad I M_{I} n \rightarrow n
$$

With new symbols defined and discussed below, we thus obtain the equations 


$$
\begin{aligned}
\mathcal{E}_{i} V_{i}(\alpha n)= & \left(\epsilon_{a}^{\prime}-E_{\bar{n}}^{*}\right) V_{i}(\alpha n)+F_{\alpha \alpha^{\prime} \beta^{\prime} \beta}\left[V_{i^{\prime}}^{*}\left(\beta n^{\prime}\right) V_{i^{\prime}}\left(\beta^{\prime} n\right)\right] V_{i}\left(\alpha^{\prime} n^{\prime}\right) \\
& +G_{\alpha \bar{\alpha}^{\prime} \beta \bar{\beta}^{\prime}}\left[U_{i^{\prime}}^{*}\left(\beta^{\prime} n^{\prime}\right) V_{i^{\prime}}(\beta n)\right] U_{i}\left(\alpha^{\prime} n^{\prime}\right), \\
\mathcal{E}_{i} U_{i}(\alpha n)= & \left(-\epsilon_{a}^{\prime \prime}-E_{\underline{n}}^{*}\right) U_{i}(\alpha n)-F_{\bar{\beta}^{\prime} \bar{\beta}^{\prime} \bar{\alpha}^{\prime} \bar{\alpha}}\left[U_{i^{\prime}}^{*}\left(\beta n^{\prime}\right) U_{i^{\prime}}\left(\alpha^{\prime} n\right)\right] U_{i}\left(\beta^{\prime} n^{\prime}\right) \\
& +G_{\bar{\alpha} \alpha^{\prime} \bar{\beta}^{\prime} \beta}\left[V_{i^{\prime}}^{*}\left(\beta n^{\prime}\right) U_{i^{\prime}}\left(\beta^{\prime} n\right)\right] V_{i}\left(\alpha^{\prime} n^{\prime}\right) .
\end{aligned}
$$

In the definitions that follow, we understand that $E_{i}$ is the energy of the state $|i\rangle$ and that $E_{\bar{n}}$ and $E_{\underline{n}}$ are, correspondingly the energies of the neighboring even states, with the subscript 0 standing either for the ground state, or for the lowest energy state considered, which for conciseness we shall continue to refer to as the ground state. We thus encounter the quantities

$$
\begin{aligned}
\mathcal{E}_{i} & =-E_{i}+\frac{1}{2}\left(E_{\overline{0}}+E_{\underline{0}}\right), \\
\epsilon_{a}^{\prime} & =h_{a}^{\prime}-\lambda, \\
\lambda & =\frac{1}{2}\left(E_{\overline{0}}-E_{\underline{0}}\right), \\
E_{n}^{*} & =E_{n}-E_{0} .
\end{aligned}
$$

The physical significance of the quantities defined in Eqs. (2.14)-(2.17) is evident. $\mathcal{E}_{i}$ are the negatives of the energies of the odd nucleus relative to the ground-state energies of its even neighbors, $\epsilon_{a}$, variously primed, are single-particle energies measured relative to the chemical potential $\lambda$, and $E_{n}^{*}$ are excitation energies of the appropriate even nuclei. Finally in achieving the form of Eq. (2.13), we have assumed that $F$ and $G$ are real. Given the Hamiltonian (2.1), Eqs. (2.12) and (2.13) are an exact set of consequences that define a non-linear eigenvalue problem with eigenvalue $\mathcal{E}_{i}$. The elements on the right hand sides of these equations define an effective Hamiltonian that will be discussed in considerable further detail in the course of this work.

We display next a functional, $\mathcal{F}$, whose vanishing first variations yield the equations of motion, namely,

$$
\begin{aligned}
\mathcal{F}= & \epsilon_{a}^{\prime}\left|V_{i}(\alpha n)\right|^{2}-\epsilon_{a}^{\prime \prime}\left|U_{i}(\alpha n)\right|^{2} \\
& \frac{1}{2} F_{\alpha \alpha^{\prime} \beta^{\prime} \beta}\left[V_{i^{\prime}}^{*}\left(\beta n^{\prime}\right) V_{i^{\prime}}\left(\beta^{\prime} n\right)\right]\left[V_{i}^{*}(\alpha n) V_{i}\left(\alpha^{\prime} n^{\prime}\right)\right] \\
& +G_{\alpha \bar{\alpha}^{\prime} \beta^{\prime} \bar{\beta}}\left[U_{i^{\prime}}^{*}\left(\beta n^{\prime}\right) V_{i^{\prime}}\left(\beta^{\prime} n\right)\right]\left[V_{i}^{*}(\alpha n) U_{i}\left(\alpha^{\prime} n^{\prime}\right)\right] \\
& -\frac{1}{2} F_{\bar{\beta} \bar{\alpha}^{\prime} \bar{\beta}^{\prime} \bar{\alpha}}\left[U_{i^{\prime}}^{*}\left(\beta n^{\prime}\right) U_{i^{\prime}}\left(\beta^{\prime} n\right)\right]\left[U_{i}^{*}(\alpha n) U_{i}\left(\alpha^{\prime} n^{\prime}\right)\right. \\
& -\mathcal{E}_{i}\left[\left|V_{i}(\alpha n)\right|^{2}+\left.U_{i}(\alpha n)\right|^{2}\right] \\
& -E_{\bar{n}}^{*}\left|V_{i}(\alpha n)\right|^{2}-E_{\underline{n}}^{*}\left|U_{i}(\alpha n)\right|^{2} \\
& \equiv \mathcal{G}-\mathcal{E}_{i}\left[\left|V_{i}(\alpha n)\right|^{2}+\left|U_{i}(\alpha n)\right|^{2}\right] .
\end{aligned}
$$

One verifies that the equations of motion (2.12) and (2.13) emerge, respectively, from the requirements

$$
\frac{\delta \mathcal{F}}{\delta V_{i}^{*}(\alpha n)}=\frac{\delta \mathcal{F}}{\delta U_{i}^{*}(\alpha n)}=0
$$


It is natural to inquire at this point if the functional $\mathcal{F}$ has any simple physical significance, in particular, if it is related to a Rayleigh-Ritz principle. To answer this question, we evaluate the sum

$$
\operatorname{Tr}(\overline{\mathrm{H}}+\underline{\mathrm{H}})=\sum_{\mathrm{n}}[\langle\overline{\mathrm{n}}|\mathrm{H}| \overline{\mathrm{n}}\rangle+\langle\underline{\mathrm{n}}|\mathrm{H}| \underline{\mathrm{n}}\rangle] .
$$

The evaluation of this sum with the aim of eventually recognizing the relevant pieces of $\mathcal{F}$ requires, in addition to the standard tool of completeness, some algebraic rearrangement of the trace involving the lighter system, just as was necessary in the equations of motion. We then find that the interaction terms match exactly those in Eq. (2.18), but that the single particle terms do not. Instead we find

$$
\begin{aligned}
h_{a}^{\prime} \rightarrow h_{a} & \equiv \bar{h}_{a}, \\
h_{a}^{\prime \prime \prime} \rightarrow h_{a} & +2 \frac{2 L+1}{2 j_{a}+1} G_{a c a c}(L)+\sqrt{\frac{2 j_{b}+1}{2 j_{a}+1}} F_{a a b b}(0) \equiv \underline{h}_{a} .
\end{aligned}
$$

We are thus tempted to replace the functional $\mathcal{F}$, as basis for the theory, by a functional that contains the new single-particle energies. We do not make this change because it destroys the simple physical significance of the Lagrange multiplier terms in Eq. (2.18) to which we next turn our attention. In practice, these extra single-particle terms are often ignored anyway.

We consider then the Lagrange-multiplier terms that appear in Eq. (2.18). The relevant question concerns the constraints that have been imposed on the variations. Since

$$
\sum_{i \alpha}\left|V_{i}(\alpha n)\right|^{2}=\sum_{\alpha}\left\langle\bar{n}\left|a_{\alpha}^{\dagger} a_{\alpha}\right| \bar{n}\right\rangle=\langle\bar{n}|\hat{N}| \bar{n}\rangle,
$$

where $\hat{N}$ is the number operator, we see that the excitation energies $E_{\bar{n}}^{*}$ enter as Lagrange multipliers for the conservation of nucleons in the heavier even nucleus. Similarly the term involving the sum over the $\left|U_{i}(\alpha n)\right|^{2}$ expresses (to an additive constant) the conservation of nucleons in the lighter system. Finally, we show that the eigenvalue $\mathcal{E}_{i}$ is (no surprise here) a Lagrange multiplier for an appropriate normalization condition. To see this we take the matrix element in the state $|i\rangle$ of the summed anticommutator,

$$
\sum_{\alpha}\left\{a_{\alpha}, a_{\alpha}^{\dagger}\right\}=\Omega, \quad \Omega=\sum_{j_{a}}\left(2 j_{a}+1\right)=\sum_{a} \Omega_{a},
$$

and thus find

$$
\frac{1}{\Omega} \sum_{\alpha n}\left[\left|V_{i}(\alpha n)\right|^{2}+\left|U_{i}(\alpha n)\right|^{2}\right]=1 .
$$

Orthogonality constraints on the solutions need not be imposed, since they follow directly from the equations of motion.

There is more to the story, however. We must note that Eq. (2.26) is only a sum of required normalization conditions. From the summed anticommutator for each level,

$$
\sum_{m_{a}}\left\{a_{\alpha}, a_{\alpha}^{\dagger}\right\}=\Omega_{a},
$$


we have

$$
\frac{1}{\Omega_{a}} \sum_{m_{a} n}\left[\left|V_{i}(\alpha n)\right|^{2}+\left.U_{i}(\alpha n)\right|^{2}\right]=1
$$

If Eqs. (2.12) and (2.13) described a linear eigenvalue problem, it would be impossible to impose the additional normalization conditions represented by Eq. (2.28). For the general non-linear problem, there is no a priori inconsistency; the satisfaction of these conditions will be a part of any fully satisfactory algorithm. The form of the normalization condition (2.28) suggests, furthermore, that it may be both useful and natural to rescale the CFP,

$$
\begin{aligned}
& V_{i}(\alpha n)=\sqrt{2 j_{a}+1} v_{i}(\alpha n), \\
& U_{i}(\alpha n)=\sqrt{2 j_{a}+1} u_{i}(\alpha n) .
\end{aligned}
$$

There is considerably more to the formal theory than what has been presented thus far. However, we have all the tools needed for the further development in the text and thus relegate the additional theoretical considerations to Appendix A.

\section{DERIVATION OF PARTICLE-ROTOR MODEL AND ITS CRANKING LIMIT: AXIALLY SYMMETRIC CASE}

As a first illustration of the formalism presented in the previous section, we assume that the even (core) nuclei are in a single axially-symmetric band $\left|I M_{I} K\right\rangle$, where $K$ is the component of the angular momentum along the figure axis. There are at least two cases where it makes some physical sense to isolate a single $K$ value, where it is the ground-state band with $K=0$, or where the band has a large $K$-value and we are dealing with an isomeric state.

We first use rotational invariance to study the structure of the amplitudes $V$ and $U$ defined in Eqs. (2.9) and (2.10), respectively. For this purpose we introduce a complete set of states $|R\rangle$ localized in the Euler angles, $R=(\alpha \beta \gamma)$, where $\alpha, \beta$ are the usual polar and azimuthal angles, respectively, and write

$$
\begin{aligned}
\left|I M_{I} K\right\rangle & =\int d R|R\rangle\left\langle R \mid I M_{I} K\right\rangle \\
& =\left(\frac{2 I+1}{8 \pi^{2}}\right)^{\frac{1}{2}} \int d R|R\rangle D_{M_{I} K}^{(I)}(R) .
\end{aligned}
$$

The identification of a scalar product of many-body states with the Wigner $D$ function is not a trivial statement, but is rather an essential element in the definition of the model to be studied. In fact, the designation $|R\rangle$ for the many-body state is insufficiently detailed and is made more explicit by the statement

$$
|R\rangle=U(R)|\hat{0} K\rangle
$$

where $|\hat{0} K\rangle$ is an axially symmetric intrinsic state spinning with angular momentum $K$ about its symmetry axis, and $U(R)$ is the unitary rotation operator in the many-body space 
defined by the Euler angles that specify the rotation $R$. For such a state, we thus note the relation, with $(\alpha \beta \gamma)=(\hat{n} \gamma)$

$$
U(\hat{n} \gamma)|\hat{0} K\rangle \exp (-i K \gamma)=U(\hat{n} 0)|0 K\rangle .
$$

The introduction of strictly localized states is, of course, an idealization that ignores the reality of band termination, but it is a standard approximation for well-deformed nuclei.

[The previous discussion and that which follows does not take into account $\mathcal{R}$ invariance, the invariance of the quadrupole shape under a rotation of $\pi$ about a principal axis. To include this symmetry in the discussion, we replace the state $\left|I M_{I} K\right\rangle$ by an eigenfunction of $\mathcal{R}$,

$$
\left.\mid I M_{I} K\right]=\frac{1}{2}\left\{\left|I M_{I} K\right\rangle+(-1)^{I+K}\left|I M_{I}-K\right\rangle\right\} .
$$

We then imitate the arguments starting on p. 8 of [1]. The task is to sort and collect the extra terms that appear both in the equations of motion and in formulas for one and two-particle observables. ]

When Eq. (3.1) is substituted into the definition (2.9) of $V$, and use is made of the definitions to be given below, we are thereby led to the study of an amplitude such as

$$
\begin{aligned}
\left\langle J M \nu\left|a_{\alpha}\right| R\right\rangle & =\left\langle J M \nu\left|U(R) U^{-1}(R) a_{\alpha} U(R)\right| \hat{0} K\right\rangle \\
& =\sum_{M^{\prime}}\left\langle J M \nu|U| J M^{\prime} \nu\right\rangle\left\langle J M^{\prime} \nu\left|U^{-1} a_{\alpha} U\right| \hat{0} K\right\rangle \\
& =\sum_{M^{\prime} \kappa_{a}} D_{M M^{\prime}}^{(J) *}(R) D_{m_{a} \kappa_{a}}^{\left(j_{a}\right) *}(R) \chi_{J M^{\prime} \nu}\left(j_{a} \kappa_{a}, K\right)(-1)^{j_{a}+\kappa_{a}},
\end{aligned}
$$

where the previous manipulations have utilized the following relations and definitions (of which the first two are standard):

$$
\begin{aligned}
\left\langle J M|U(R)| J M^{\prime}\right\rangle & =D_{M M^{\prime}}^{(J) *}(R), \\
U^{-1}(R) a_{j m} U(R) & =\sum_{\kappa} a_{j \kappa} D_{m \kappa}^{(j) *}(R), \\
\left\langle J M \nu\left|a_{j m}\right| \hat{0}\right\rangle & \equiv(-1)^{j+m} \chi_{J M \nu}(j m, K)
\end{aligned}
$$

The phase in (3.8) has been introduced for algebraic convenience.

With the help of the integral of a product of three $D$ functions ( [37], Eq. (4.6.2)) and the application of standard symmetry properties of CG coefficients (Eqs. (3.5.15) and (3.5.16) of the same reference), we find for the CFP defined in Eq. (2.9),

$$
\begin{aligned}
V_{J M \nu}\left(\alpha I M_{I} K\right)= & \sum_{\kappa_{a}} \sqrt{\frac{8 \pi^{2}}{2 j_{a}+1}}(-1)^{J-M} \\
& \times\left(I M_{I} J-M \mid j_{a} m_{a}\right)\left(J K-\kappa_{a} j_{a} \kappa_{a} \mid I K\right) \\
& \times(-1)^{j_{a}+\kappa_{a}} \chi_{J K-\kappa_{a} \nu}\left(j_{a} \kappa_{a}, K\right) .
\end{aligned}
$$

A similar analysis carried out for the amplitude $U$ defined in (2.10) yields the result

$$
\begin{aligned}
U_{J M \nu}\left(\alpha I M_{I} K\right)= & \sum_{\kappa_{a}} \sqrt{\frac{8 \pi^{2}}{2 j_{a}+1}} \\
& \times(-1)^{J-M+j_{a}-\kappa_{a}+j_{a}+m_{a}}\left(I M_{I} J-M \mid j_{a} m_{a}\right) \\
& \times\left(J K-\kappa_{a} j_{a} \kappa_{a} \mid I K\right) \phi_{J K-\kappa_{a} \nu}\left(j_{a} \kappa_{a}, K\right), \\
\phi_{J M \nu}\left(j_{a} \kappa_{a}, K\right)= & \left\langle J M \nu\left|a_{j_{a}-\kappa_{a}}^{\dagger}\right| \hat{0} K\right\rangle .
\end{aligned}
$$


It is most succinct to base further discussion on the variational principle (2.18). We evaluate this expression when the core collective states are restricted to the members of a single band of an axial rotor, and the states of the odd nucleus are any states that can arise from the coupling. Returning to a full nomenclature, this calls for the identifications

$$
\begin{aligned}
& \bar{n} \rightarrow \overline{I M_{I} K}, \quad \underline{n} \rightarrow \underline{I M_{I} K}, \\
& i \rightarrow J M \nu .
\end{aligned}
$$

We are assuming here that there are corresponding bands in the two even nuclei that couple to the given odd nucleus. In the following we shall also suppress the bar and underline in the CFP, understanding them from context, but continue to emphasize this distinction in the excitation energies $E^{*}$.

We consider first the evaluation of all terms in the variational principle depending on $\left|V_{i}(\alpha n)\right|^{2}$, which includes some of the single-particle terms and some of the Lagrangemultiplier terms. Writing $\bar{E}^{*}(I K)$ for $E_{\bar{n}}^{*}$, we consider in particular the combination

$$
-\sum\left(\mathcal{E}_{J \nu}+\bar{E}^{*}(I K)\right)\left|V_{J M \nu}\left(\alpha I M_{I} K\right)\right|^{2}
$$

For the evaluation of the CFP $V$, we utilize Eq. (3.9), renormalized, however, by a factor of $\left(1 / \sqrt{8 \pi^{2}}\right)$ in order that the reciprocal of such factors do not appear in the answer, i. e., we rescale $\chi$ by this factor. For the evaluation of expressions involving the CFP $U$, we shall utilize Eq. (3.10) similarly renormalized. This rescaling will be understood throughout the remainder of this paper.

The rest of this section consists of a relatively detailed account of the evaluation of the variational sum in the "intrinsic" system. Subsequent variation will led to the self-consistent version of the strong coupling PRM. Toward this end, as part of the definition of an axial rotor, we assume that, equally for the barred and underlined quantities,

$$
E^{*}(I K) \rightarrow E^{*}\left(\vec{I}^{2}-K^{2}\right)=E^{*}\left(\hat{I}_{1}^{2}+\hat{I}_{2}^{2}+\hat{I}_{3}^{2}-K^{2}\right),
$$

where we have introduced intrinsic components of the angular momentum. The arrow indicates the replacement of an eigenvalue by an operator. This is done by making use of the appropriate one of the CG coefficients, understood as a scalar product, that appear in Eq. (2.9), as follows

$$
E^{*}(I K)\left(J K-\kappa_{a} j_{a} \kappa_{a} \mid I K\right)=\left(J K-\kappa_{a} j_{a} \kappa_{a}\left|E^{*}\left(\vec{I}^{2}-K^{2}\right)\right| I K\right) .
$$

Furthermore, in each term of the sum $I$ is coupled with some $j_{a}$ to a value of $J$. From the structure of the CFP, it follows that we may replace $\vec{I}$ by $\vec{J}+\vec{j}_{a}$ in Eq. (3.15) and write (with $j_{a} \rightarrow j$ ),

$$
\begin{aligned}
\bar{E}^{*}\left(\vec{I}^{2}-K^{2}\right) & =\bar{E}^{*}\left[(\vec{J}+\vec{j})^{2}-K^{2}\right] \\
& =\bar{E}^{*}\left(\vec{J}^{2}-K^{2}\right)+\frac{\partial \bar{E}^{*}}{\partial \hat{J}_{i}} \hat{j}_{i}+\frac{1}{2} \frac{\partial^{2} \bar{E}^{*}}{\partial \hat{J}_{i}^{2}}+\ldots .
\end{aligned}
$$

It is not necessary for these considerations that $E(I K)$ have the simple form of a rotor spectrum, only that it be a function as indicated. The first term of Eq. (3.16) may be 
replaced by an eigenvalue $\left.\bar{E}^{*}\left[J(J+1)-K^{2}\right)\right]$, and the second term, the Coriolis coupling will be evaluated below. The third and possible higher order terms will not be studied here, but will be included in applications. Other than the Coriolis coupling and higher-order terms, the contribution from Eq. (3.13) and of the remaining single-particle terms takes the form

$$
\begin{aligned}
& \sum_{J \nu j_{a} \kappa_{a}}\left(\epsilon_{a}^{\prime}-\bar{\varepsilon}_{J \nu}\right)\left|\chi_{J K-\kappa_{a} \nu}\left(j_{a} \kappa_{a}, K\right)\right|^{2}, \\
& \bar{\varepsilon}_{J \nu}=\mathcal{E}_{J \nu}-\bar{E}^{*}\left[J(J+1)-K^{2}\right] .
\end{aligned}
$$

For the further evaluation we can write in full generality

$$
\frac{\partial E^{*}}{\partial J_{i}} \equiv f\left(\vec{J}^{2}-K^{2}\right) J_{i}
$$

where in the simplest case $f$ is just the reciprocal of the moment of inertia. Using the matrix elements of the raising and lowering operators, and collecting terms, we find, in connection with the second term of Eq. (3.16), the Coriolis coupling term, the following sum to evaluate

$$
\begin{aligned}
& -\frac{1}{2} \sum_{M_{I} m_{a} M I J \kappa_{a} \kappa_{a}^{\prime}} \chi_{J K-\kappa_{a} \nu}\left(j_{a} \kappa_{a}, K\right) \chi_{J K-\kappa_{a}^{\prime} \nu}^{*}\left(j_{a} \kappa_{a}^{\prime}, K\right)\left(2 j_{a}+1\right)^{-1} \\
& \times(-1)^{2 j_{a}+\kappa_{a}+\kappa_{a}^{\prime}}\left|\left(I M_{I} J-M \mid j_{a} m_{a}\right)\right|^{2}\left(J K-\kappa_{a}^{\prime} j_{a} \kappa_{a}^{\prime} \mid I K\right) \\
& \times \bar{f}(J K)\left[\sqrt{\left(J-K+\kappa_{a}\right)\left(J+K-\kappa_{a}+1\right)}\right. \\
& \sqrt{\left(j_{a}+\kappa_{a}\right)\left(j_{a}-\kappa_{a}+1\right)}\left(J K-\kappa_{a}+1 j_{a} \kappa_{a}-1 \mid I K\right) \\
& +\sqrt{\left(J+K-\kappa_{a}\right)\left(J-K+\kappa_{a}+1\right)} \sqrt{\left(j_{a}-\kappa_{a}\right)\left(j_{a}+\kappa_{a}+1\right)} \\
& \left.\times\left(J K-\kappa_{a}-1 j_{a} \kappa_{a}+1 \mid I K\right)+2\left(K-\kappa_{a}\right) \kappa_{a}\left(J K-\kappa_{a} j_{a} \kappa_{a} \mid I K\right)\right] \text {. }
\end{aligned}
$$

With the help of the standard orthonormalization conditions for CG coefficients this reduces to the final result for the term under study,

$$
\begin{aligned}
& -\frac{1}{2} \sum_{J \nu j_{a} \kappa_{a}} \chi_{J K-\kappa_{a} \nu}^{*}\left(j_{a} \kappa_{a}, K\right) \bar{f}(J K) \\
& \times\left[\sqrt{\left(J+K-\kappa_{a}\right)\left(J-K+\kappa_{a}+1\right)} \sqrt{\left(j_{a}-\kappa_{a}\right)\left(j_{a}+\kappa_{a}+1\right)}\right. \\
& \times \chi_{J K-\kappa_{a}-1 \nu}\left(j_{a} \kappa_{a}+1, K\right) \\
& +\sqrt{\left(J-K+\kappa_{a}\right)\left(J+K-\kappa_{a}+1\right)} \sqrt{\left(j_{a}+\kappa_{a}\right)\left(j_{a}-\kappa_{a}+1\right)} \\
& \times \chi_{J K-\kappa_{a}+1 \nu}\left(j_{a} \kappa_{a}-1, K\right) \\
& \left.+2\left(K-\kappa_{a}\right) \kappa_{a} \chi_{J K-\kappa_{a} j_{a} \kappa_{a}}\left(j_{a} \kappa_{a}, K\right)\right] \text {. }
\end{aligned}
$$

We outline briefly the corresponding calculation of the single-particle terms associated with the $U$ coefficients. Only the following change is necessary: All barred energies associated with the heavier of the two neighboring even nuclei are replaced by underlined energies associated with the lighter of the two neighbors. For the terms corresponding to Eq. 3.17), we find 


$$
-\sum_{J \nu j_{a} \kappa_{a}}\left(\epsilon_{a}^{\prime \prime}+\underline{\varepsilon}_{J \nu}\right)\left|\phi_{J K-\kappa_{a} \nu}\left(j_{a} \kappa_{a}, K\right)\right|^{2}
$$

For the Coriolis coupling term, we find

$$
\begin{aligned}
& \frac{1}{2} \sum_{J \nu j_{a} \kappa_{a}} \phi_{J K-\kappa_{a} \nu}^{*}\left(j_{a} \kappa_{a}, K\right) \underline{f}(J K) \\
& \times\left[\sqrt{\left(J+K-\kappa_{a}\right)\left(J-K+\kappa_{a}+1\right)} \sqrt{\left(j_{a}-\kappa_{a}\right)\left(j_{a}+\kappa_{a}+1\right)}\right. \\
& \times \phi_{J K-\kappa_{a}-1 \nu}\left(j_{a} \kappa_{a}+1, K\right) \\
& +\sqrt{\left(J-K+\kappa_{a}\right)\left(J+K-\kappa_{a}+1\right)} \sqrt{\left(j_{a}+\kappa_{a}\right)\left(j_{a}-\kappa_{a}+1\right)} \\
& \left.\times \phi_{J K-\kappa_{a}+1 \nu}\left(j_{a} \kappa_{a}-1, K\right)+\left(K-\kappa_{a}\right) \kappa_{a} \phi_{J K-\kappa_{a} \nu}\left(j_{a} \kappa_{a}, K\right)\right]
\end{aligned}
$$

We turn to the contribution of the interaction terms. We evaluate a typical quartic contribution to Eq. (2.20), for example

$$
\begin{aligned}
& \sum_{\chi} \equiv \frac{1}{2} F_{\alpha \alpha^{\prime} \beta^{\prime} \beta} V_{J M \nu}\left(\beta^{\prime} I M_{I} K\right) V_{J M \nu}^{*}\left(\beta I^{\prime} M_{I^{\prime}} K\right) \\
& \times V_{J^{\prime} M^{\prime} \nu^{\prime}}\left(\alpha^{\prime} I^{\prime} M_{I^{\prime}} K\right) V_{J^{\prime} M^{\prime} \nu^{\prime}}^{*}\left(\alpha I M_{I} K\right) \\
& =F_{a a^{\prime} b^{\prime} b}(L)\left(j_{a} m_{a} j_{a^{\prime}}-m_{a^{\prime}} \mid L m_{a}-m_{a^{\prime}}\right)\left(j_{b^{\prime}} m_{b^{\prime}} j_{b}-m_{b} \mid L m_{b^{\prime}}-m_{b}\right) \\
& \times(-1)^{j_{a^{\prime}}}-m_{a^{\prime}}+j_{b}-m_{b}(-1)^{j_{a}+\kappa_{a}+j_{a^{\prime}}+\kappa_{a^{\prime}}+j_{b}+\kappa_{b}+j_{b^{\prime}}+\kappa_{b^{\prime}}} \\
& \times\left[\left(2 j_{a}+1\right)\left(2 j_{a^{\prime}}+1\right)\left(2 j_{b}+1\right)\left(2 j_{b^{\prime}}+1\right)\right]^{-\frac{1}{2}} \\
& \times \chi_{J K-\kappa_{b^{\prime}}}\left(j_{b^{\prime}} \kappa_{b^{\prime}}\right) \chi_{J K-\kappa_{b} \nu}^{*}\left(j_{b} \kappa_{b}\right) \chi_{J^{\prime} K-\kappa_{a^{\prime} \nu^{\prime}}}\left(j_{a^{\prime}} \kappa_{a^{\prime}}\right) \chi_{J^{\prime} K-\kappa_{a} \nu^{\prime}}^{*}\left(j_{a} \kappa_{a}\right) \\
& \times\left(I M_{I} J m_{b^{\prime}}-M_{I} \mid j_{b^{\prime}} m_{b^{\prime}}\right)\left(I^{\prime} M_{I^{\prime}} J m_{b}-M_{I^{\prime}} \mid j_{b} m_{b}\right) \\
& \times\left(I^{\prime} M_{I^{\prime}} J^{\prime} m_{a^{\prime}}-M_{I^{\prime}} \mid j_{a^{\prime}} m_{a^{\prime}}\right)\left(I M_{I} J^{\prime} m_{a}-M_{I} \mid j_{a} m_{a}\right) \\
& \times\left(J K-\kappa_{b^{\prime}} j_{b^{\prime}} \kappa_{b^{\prime}} \mid I K\right)\left(J K-\kappa_{b} j_{b} \kappa_{b} \mid I^{\prime} K\right) \\
& \times\left(J^{\prime} K-\kappa_{a^{\prime}} j_{a^{\prime}} \kappa_{a^{\prime}} \mid I^{\prime} K\right)\left(J^{\prime} K-\kappa_{a} j_{a} \kappa_{a} \mid I K\right) .
\end{aligned}
$$

To obtain this form from the corresponding term of Eq. (2.18) we have utilized Eqs. (3.9) and (2.2). From angular momentum conservation, we have the relations

$$
m_{b^{\prime}}-M_{I}=m_{b}-M_{I^{\prime}}, m_{a^{\prime}}-M_{I^{\prime}}=m_{a}-M_{I} .
$$

The next step is to perform the sums over the magnetic quantum numbers $m_{a}$ and $m_{b}$. For this purpose, we use Eq. (6.6.27) of [37] with assists from Eqs. (3.5.14)-(3.5.17) of the same reference. At this point we obtain the sum (henceforth we simplify the arguments of the single-particle amplitudes, $j_{a} \kappa_{a}, K \rightarrow a$, etc.)

$$
\begin{aligned}
\sum_{\chi}= & \frac{1}{2} F_{a a^{\prime} b^{\prime} b}(-1)^{j_{a}+\kappa_{a}+\kappa_{a^{\prime}}+j_{b^{\prime}}+\kappa_{b^{\prime}}+\kappa_{b}+J+J^{\prime}}(2 L+1) \\
& \times \chi_{J K-\kappa b^{\prime} \nu}\left(b^{\prime}\right) \chi_{j K-\kappa_{b} \nu}^{*}(b) \chi_{J^{\prime} K-\kappa_{a^{\prime} \nu^{\prime}}\left(a^{\prime}\right) \chi_{J^{\prime} K-\kappa_{a} \nu^{\prime}}^{*}(a)} \\
& \times\left(J K-\kappa_{b^{\prime}} j_{b^{\prime}} \kappa_{b^{\prime}} \mid I K\right)\left(J K-\kappa_{b} j_{b} \kappa_{b} \mid I^{\prime} K\right) \\
& \times\left(J^{\prime} K-\kappa_{a^{\prime}} j_{a^{\prime}} \kappa_{a^{\prime}} \mid I^{\prime} K\right)\left(J^{\prime} K-\kappa_{a} j_{a} \kappa_{a} \mid I K\right) \\
& \times\left\{\begin{array}{ccc}
j_{a^{\prime}} & j_{a} & L \\
I & I^{\prime} & J^{\prime}
\end{array}\right\}\left\{\begin{array}{ccc}
j_{b} & j_{b^{\prime}} & L \\
I & I^{\prime} & J
\end{array}\right\} .
\end{aligned}
$$


The final summations that we expect to be able to do in general are over $I$ and $I^{\prime}$. Toward this end we need to re express the $6-j$ symbols that occur in Eq. (3.26) in terms of $6-j$ symbols that depend separately on $I$ and $I^{\prime}$. This can be done by the application of Eq. (6.2.12) of [37]. We quote the value of $\sum_{\chi}$ that results from this transformation:

$$
\begin{aligned}
& \sum_{\chi}=\frac{1}{2} \sum_{I I^{\prime} \ldots} F_{a a^{\prime} b^{\prime} b}(L)(-1)^{\kappa_{a}+\kappa_{b}+\kappa_{a^{\prime}}+\kappa_{b^{\prime}}+j_{a^{\prime}}+j_{b}+L+I+I^{\prime}+I^{\prime \prime}} \\
& \times(2 L+1)\left(2 I^{\prime \prime}+1\right)\left\{\begin{array}{ccc}
j_{a^{\prime}} & j_{a} & L \\
j_{b^{\prime}} & j_{b} & I^{\prime \prime}
\end{array}\right\}\left\{\begin{array}{ccc}
j_{a} & I & J^{\prime} \\
J & I^{\prime \prime} & j_{b^{\prime}}
\end{array}\right\}\left\{\begin{array}{ccc}
j_{a^{\prime}} & J^{\prime} & I^{\prime} \\
J & j_{b} & I^{\prime \prime}
\end{array}\right\} \\
& \times\left(J K-\kappa_{b^{\prime}} j_{b^{\prime}} \kappa_{b^{\prime}} \mid I K\right)\left(J^{\prime} K-\kappa_{a} j_{a} \kappa_{a} \mid I K\right) \\
& \times\left(J K-\kappa_{b} j_{b} \kappa_{b} \mid I^{\prime} K\right)\left(J^{\prime} K-\kappa_{a^{\prime}} j_{a^{\prime}} \kappa_{a^{\prime}} \mid I^{\prime} K\right) \\
& \times \chi_{J K-\kappa_{b^{\prime}} \nu}\left(j_{b^{\prime}} \kappa_{b^{\prime}}\right) \chi_{J K-\kappa_{b} \nu}^{*}\left(j_{b} \kappa_{b}\right) \\
& \times \chi_{J^{\prime} K-\kappa_{a^{\prime}} \nu^{\prime}}\left(j_{a^{\prime}} \kappa_{a^{\prime}}\right) \chi_{J^{\prime} K-\kappa_{a} \nu^{\prime}}^{*}\left(j_{a} \kappa_{a}\right) .
\end{aligned}
$$

We are finally in a position to do the sums over $I$ and $I^{\prime}$ by the application of Eq. (6.2.6) of [37]. This leads to our final exact result. It turns out that the evaluation of the remaining interaction terms parallels that just described, differing only in the coupling constants and the single-particle functions that occur. For economy of expression, it is this total result that we finally quote:

$$
\begin{aligned}
& \sum=\sum_{I^{\prime \prime} J J^{\prime} \nu \nu^{\prime} j_{a} \ldots \kappa_{a} \ldots}\left[\frac{1}{2} F_{a a^{\prime} b^{\prime} b}(L) \chi_{J K-\kappa_{b^{\prime}} \nu}\left(b^{\prime}\right) \chi_{J K-\kappa_{b} \nu}^{*}(b) \chi_{J^{\prime} K-\kappa_{a^{\prime}} \nu^{\prime}}\left(a^{\prime}\right) \chi_{J^{\prime} K-\kappa_{a} \nu^{\prime}}^{*}(a)\right. \\
& -\frac{1}{2} F_{b a^{\prime} b^{\prime} a}(L) \phi_{J K-\kappa_{b^{\prime} \nu}}\left(b^{\prime}\right) \phi_{J K-\kappa_{b} \nu}^{*}(b) \phi_{J^{\prime} K-\kappa_{a^{\prime}} \nu^{\prime}}\left(a^{\prime}\right) \phi_{J^{\prime} K-\kappa_{a} \nu^{\prime}}^{*}(a) \\
& \left.+G_{a a^{\prime} b^{\prime} b} \chi_{J K-\kappa_{b^{\prime}}}\left(b^{\prime}\right) \phi_{J K-\kappa_{b} \nu}^{*}(b) \phi_{J^{\prime} K-\kappa_{a^{\prime} \nu^{\prime}}}\left(a^{\prime}\right) \chi_{J^{\prime} K-\kappa_{a} \nu^{\prime}}^{*}(a)\right] \\
& \times(-1)^{\kappa_{b}+\kappa_{b^{\prime}}-1+j_{a^{\prime}}+j_{b}+L+I^{\prime \prime}} \\
& \times \frac{(2 L+1)}{\left(2 J^{\prime}+1\right)}\left\{\begin{array}{ccc}
j_{a^{\prime}} & j_{a} & L \\
j_{b^{\prime}} & j_{b} & I^{\prime \prime}
\end{array}\right\} \\
& \times\left(j_{a}-\kappa_{a} j_{b^{\prime}} \kappa_{b^{\prime}} \mid I^{\prime \prime} \kappa_{b^{\prime}}-\kappa_{a}\right)\left(j_{a^{\prime}}-\kappa_{a^{\prime}} j_{b} \kappa_{b} \mid I^{\prime \prime} \kappa_{b}-\kappa_{a^{\prime}}\right) \\
& \times\left(I^{\prime \prime} \kappa_{b^{\prime}}-\kappa_{a} J K-\kappa_{b^{\prime}} \mid J^{\prime} K-\kappa_{a}\right)\left(I^{\prime \prime} \kappa_{b}-\kappa_{a^{\prime}} J K-\kappa_{b} \mid J^{\prime} K-\kappa_{a^{\prime}}\right) \text {. }
\end{aligned}
$$

We now collect the results of the calculations presented in this section. The strong coupling limit of the functional $\mathcal{F}$ for the axial case is given by the sum of Eqs. (3.17), (3.21), (3.22), (3.23), and (3.28). By varying in turn with respect to $\chi_{J K-\kappa_{a} \nu}^{*}(a)$ and $\phi_{J K-\kappa_{a} \nu}^{*}$, we obtain the equations of motion

$$
\begin{aligned}
\bar{\varepsilon}_{J \nu} \chi_{J K-\kappa_{a} \nu}(a)= & \epsilon_{a}^{\prime} \chi_{J K-\kappa_{a} \nu}(a)-\frac{1}{2} \bar{f}(J K) \sqrt{\left(J+K-\kappa_{a}\right)\left(J-K+\kappa_{a}+1\right)} \\
& \times \sqrt{\left(j_{a}-\kappa_{a}\right)\left(j_{a}+\kappa_{a}+1\right)} \chi_{J K-\kappa_{a}-1 \nu}(a) \\
& +\sqrt{\left(J-K+\kappa_{a}\right)\left(J+K-\kappa_{a}+1\right)} \sqrt{\left(j_{a}+\kappa_{a}\right)\left(j_{a}-\kappa_{a}+1\right)} \chi_{J K-\kappa_{a}+1 \nu}(a) \\
& +2\left(K-\kappa_{a}\right) \kappa_{a} \chi_{J K-\kappa_{a} \nu}(a) \\
& +\left[F_{a a^{\prime} b^{\prime} b}(L) \chi_{J^{\prime} K-\kappa_{b^{\prime} \nu^{\prime}}}\left(b^{\prime}\right) \chi_{J^{\prime} K-\kappa_{b} \nu^{\prime}}^{*}(b) \chi_{J K-\kappa_{a^{\prime} \nu}}\left(a^{\prime}\right)\right. \\
& \left.+G_{a a^{\prime} b^{\prime} b}(L) \chi_{J^{\prime} K-\kappa_{b^{\prime} \nu^{\prime}}}\left(b^{\prime}\right) \phi_{J^{\prime} K-\kappa_{b} \nu^{\prime}}^{*}(b) \phi_{J K-\kappa_{a^{\prime}}}\left(a^{\prime}\right)\right]
\end{aligned}
$$




$$
\begin{aligned}
& \times \frac{2 L+1}{2 J+1}\left\{\begin{array}{ccc}
j_{a^{\prime}} & j_{a} & L \\
j_{b^{\prime}} & j_{b} & I
\end{array}\right\} \\
& \times\left(j_{a}-\kappa_{a} j_{b^{\prime}} \kappa_{b^{\prime}} \mid I \kappa_{b^{\prime}}-\kappa_{a}\right)\left(j_{a^{\prime}}-\kappa_{a^{\prime}} j_{b} \kappa_{b} \mid I \kappa_{b}-\kappa_{a^{\prime}}\right) \\
& \times\left(I \kappa_{b^{\prime}}-\kappa_{a} J^{\prime} K-\kappa_{b^{\prime}} \mid J K-\kappa_{a}\right)\left(I \kappa_{b}-\kappa_{a^{\prime}} J^{\prime} K-\kappa_{b} \mid J K-\kappa_{a^{\prime}}\right) \\
\underline{\varepsilon}_{J \nu} \phi_{J K-\kappa_{a} \nu}(a)= & -\epsilon_{a}^{\prime \prime} \phi_{J K-\kappa_{a} \nu}(a)-\frac{1}{2} \underline{f}(J K) \sqrt{\left(J+K-\kappa_{a}\right)\left(J-K+\kappa_{a}+1\right)} \\
& \times \sqrt{\left(j_{a}-\kappa_{a}\right)\left(j_{a}+\kappa_{a}+1\right)} \phi_{J K-\kappa_{a}-1 \nu}(a) \\
& +\sqrt{\left(J-K+\kappa_{a}\right)\left(J+K-\kappa_{a}+1\right)} \sqrt{\left(j_{a}+\kappa_{a}\right)\left(j_{a}-\kappa_{a}+1\right)} \phi_{J K-\kappa_{a}+1 \nu}(a) \\
& +2\left(K-\kappa_{a}\right) \kappa_{a} \phi_{J K-\kappa_{a} \nu}(a) \\
& -\left[F_{b a^{\prime} b^{\prime} a}(L) \phi_{J^{\prime} K-\kappa_{b^{\prime}} \nu^{\prime}}\left(b^{\prime}\right) \phi_{J^{\prime} K-\kappa_{b} \nu^{\prime}}^{*}(b) \phi_{J K-\kappa_{a^{\prime}} \nu}\left(a^{\prime}\right)\right. \\
& \left.+G_{a a^{\prime} b^{\prime} b}(L) \phi_{J^{\prime} K-\kappa_{b^{\prime}} \nu^{\prime}}\left(b^{\prime}\right) \chi_{J^{\prime} K-\kappa_{b} \nu^{\prime}}^{*}(b) \chi_{J K-\kappa_{a^{\prime}} \nu}\left(a^{\prime}\right)\right] \\
& \times \frac{2 L+1}{2 J+1}\left\{\begin{array}{l}
j_{a^{\prime}} j_{a} L \\
j_{b^{\prime}} \quad j_{b} I
\end{array}\right\} \\
& \times\left(j_{a}-\kappa_{a} j_{b^{\prime}} \kappa_{b^{\prime}} \mid I \kappa_{b^{\prime}}-\kappa_{a}\right)\left(j_{a^{\prime}}-\kappa_{a^{\prime}} j_{b} \kappa_{b} \mid I \kappa_{b}-\kappa_{a^{\prime}}\right) \\
& \times\left(I \kappa_{b^{\prime}}-\kappa_{a} J^{\prime} K-\kappa_{b^{\prime}} \mid J K-\kappa_{a}\right)\left(I \kappa_{b}-\kappa_{a^{\prime}} J^{\prime} K-\kappa_{b} \mid J K-\kappa_{a^{\prime}}\right)
\end{aligned}
$$

We add the normalization conditions for the particle-rotor model that follow from Eq. (2.28). We find

$$
\sum_{\kappa_{a}}\left[\left|\chi_{J K-\kappa_{a} \nu}(a)\right|^{2}+\left|\phi_{J K-\kappa_{a} \nu}(a)\right|^{2}\right]=2 j_{a}+1 .
$$

\section{ALTERNATIVE DERIVATION AND ITS CRANKING LIMIT:AXIAL CASE}

In addition to the PRM, self-consistent or otherwise, we are interested in the cranking theory, valid in the limit in which a single-particle angular momentum $j_{a}$ may be neglected compared to the collective angular momentum. In principle, we should be able to derive this limit from the form of the theory developed in Sec. II. However, the interaction terms, as derived, do not provide a natural pathway to the limit sought. Therefore we start anew in this section, but concentrate on deriving an approximate version of the PRM in which an expansion in $(\langle j\rangle / J)$ has been made, the main difference compared to the previous calculation residing in the treatment of the interaction terms. We derive an approximate version of the PRM and then introduce the additional approximation necessary to reach the cranking limit.

For present purposes it is convenient to work in coordinate-spin-isospin space, designated by $x$. We work with amplitudes that we refer to as coordinate coefficients of fractional parentage (CCFP),

$$
\begin{aligned}
V_{J M \nu}\left(x I M_{I} K\right) & =\left\langle J M \nu|\hat{\psi}(x)| \overline{I M_{I} K}\right\rangle, \\
U_{J M \nu}\left(x I M_{I} K\right) & =\left\langle J M \nu\left|\hat{\psi}^{\dagger}(x)\right| \underline{I M_{I} K}\right\rangle,
\end{aligned}
$$

where $\hat{\psi}(x)$ is the nucleon destruction operator at the space-spin-isospin point $x$. In terms of these amplitudes, we rewrite the variational functional $\mathcal{F}$ of Eq. (2.19) as 


$$
\begin{aligned}
\mathcal{F}= & {\left[\epsilon\left(x x^{\prime}\right)-\mathcal{E}_{J \nu} \delta\left(x-x^{\prime}\right)-\bar{E}^{*}(I K) \delta\left(x-x^{\prime}\right)\right] V_{J M \nu}\left(x^{\prime} I M_{I} K\right) V_{J M \nu}^{*}\left(x I M_{I} K\right) } \\
& {\left[-\epsilon\left(x x^{\prime}\right)-\mathcal{E}_{J \nu} \delta\left(x-x^{\prime}\right)-\underline{E}^{*}(I K) \delta\left(x-x^{\prime}\right)\right] U_{J M \nu}\left(x I M_{I} K\right) U_{J M \nu}^{*}\left(x^{\prime} I M_{I} K\right) } \\
& +\frac{1}{2} F\left(x x^{\prime} x^{\prime \prime} x^{\prime \prime \prime}\right) V_{J^{\prime} M^{\prime} \nu^{\prime}}\left(x^{\prime \prime} I M_{I} K\right) V_{J^{\prime} M^{\prime} \nu^{\prime}}^{*}\left(x^{\prime \prime \prime} I^{\prime} M_{I^{\prime}} K\right) \\
& \times V_{J M \nu}\left(x^{\prime} I^{\prime} M_{I^{\prime}} K\right) V_{J M \nu}^{*}\left(x I M_{I} K\right) \\
& +G\left(x x^{\prime} x^{\prime \prime} x^{\prime \prime \prime}\right) V_{J^{\prime} M^{\prime} \nu^{\prime}}\left(x^{\prime \prime} I M_{I} K\right) U_{J^{\prime} M^{\prime} \nu^{\prime}}^{*}\left(x^{\prime \prime \prime} I^{\prime} M_{I^{\prime}} K\right) \\
& \times U_{J M \nu}\left(x^{\prime} I^{\prime} M_{I^{\prime}} K\right) V_{J M \nu}^{*}\left(x I M_{I} K\right) \\
& -\frac{1}{2} F\left(x^{\prime \prime \prime} x^{\prime} x^{\prime \prime} x\right) U_{J^{\prime} M^{\prime} \nu^{\prime}}\left(x^{\prime \prime} I M_{I} K\right) V U_{J^{\prime} M^{\prime} \nu^{\prime}}^{*}\left(x^{\prime \prime \prime} I^{\prime} M_{I^{\prime}} K\right) \\
& \times U_{J M \nu}\left(x^{\prime} I^{\prime} M_{I^{\prime}} K\right) U_{J M \nu}^{*}\left(x I M_{I} K\right) .
\end{aligned}
$$

We have set $\epsilon^{\prime}=\epsilon^{\prime \prime}=\epsilon$ and shall adhere to this simplification for the remainder of our presentation. To carry out the transformation to Eq. (1.3), we have made use of a special mode transformation to a basis in which $\epsilon\left(x x^{\prime}\right)$ is diagonal,

$$
\begin{aligned}
a_{\alpha} & =\varphi_{\alpha}^{*}(x) \hat{\psi}(x), \\
\epsilon_{a} \varphi_{\alpha}(x) & =\epsilon\left(x x^{\prime}\right) \varphi_{\alpha}\left(x^{\prime}\right), \\
F\left(x x^{\prime} x^{\prime \prime} x^{\prime \prime \prime}\right) & =F_{\alpha \gamma \delta \beta} \varphi_{\alpha}^{*}(x) \varphi_{\gamma}\left(x^{\prime}\right) \varphi_{\delta}^{*}\left(x^{\prime \prime}\right) \varphi_{\beta}\left(x^{\prime \prime \prime}\right), \\
G\left(x x^{\prime} x^{\prime \prime} x^{\prime \prime \prime}\right) & =G_{\alpha \gamma \beta \delta} \varphi_{\delta}^{*}\left(x^{\prime \prime \prime}\right) \varphi_{\beta}^{*}\left(x^{\prime}\right) \varphi_{\gamma}\left(x^{\prime}\right) \varphi_{\alpha}(x) .
\end{aligned}
$$

The major device of the present derivation is to transform from angular momentum eigenfunctions to eigenfunctions localized in angle space, a technique that has already been exploited in Sec. IV. We base the developments on expressions for the CCFP that are derived by the same initial transformations that led to Eqs. (3.9) and (3.10), namely

$$
\begin{aligned}
\left(\begin{array}{c}
V_{J M \nu}\left(x M_{I} K\right) \\
U_{J M \nu}\left(x M_{I} K\right)
\end{array}\right) & =\int d R D_{M M^{\prime}}^{(J) *}(R)\left(\begin{array}{c}
\chi_{J M^{\prime} \nu}(R x, K) \\
\phi_{J M^{\prime} \nu}(R x, K)
\end{array}\right) \sqrt{\frac{2 I+1}{8 \pi^{2}}} D_{M_{I} K}^{(I)}(R), \\
\chi_{J M \nu}(R x, K) & =\langle J M \nu|\hat{\psi}(R x)| \hat{0} K\rangle, \\
\phi_{J M \nu}(R x, K) & =\left\langle J M \nu\left|\hat{\psi}^{\dagger}(R x)\right| \hat{0} K\right\rangle, \\
\hat{\psi}(R x) & =U^{-1}(R) \hat{\psi}(x) U(R) .
\end{aligned}
$$

When we substitute Eq. (4.8) into the first two terms of Eq. (4.3), we encounter the restricted completeness relation

$$
\sum_{I M} D_{M K}^{(I)}(R) D_{M K}^{(I) *}\left(R^{\prime}\right) \frac{2 I+1}{8 \pi^{2}}=\delta\left(\hat{n}-\hat{n}^{\prime}\right) \exp \left[-i K\left(\gamma-\gamma^{\prime}\right)\right] \frac{1}{2 \pi} .
$$

To perform the integral over $\gamma^{\prime}$, we note the relation (3.3), and in the further calculation we utilize basic properties of the $D$ functions, following from their definition, Eq. (3.6), the first one also used extensively for the interaction term to be computed below,

$$
\begin{aligned}
\sum_{M} D_{M^{\prime} M}^{(J)}\left(R^{-1}\right) D_{M M^{\prime \prime}}^{(J)}\left(R^{\prime}\right) & =D_{M^{\prime} M^{\prime \prime}}^{(J)}\left(R^{-1} R^{\prime}\right), \\
D_{M M^{\prime}}^{(J)}(0) & =\delta_{M, M^{\prime}} .
\end{aligned}
$$


Finally, invoking the rotational invariance of the Hamiltonian, which for the term under consideration means that $\epsilon\left(R x R x^{\prime}\right)=\epsilon\left(x x^{\prime}\right)$, we obtain the result (recall that factors of $8 \pi^{2}$ are suppressed)

$$
\left[\epsilon\left(x x^{\prime}\right)-\mathcal{E}_{J \nu} \delta\left(x-x^{\prime}\right)\right] \chi_{J M \nu}\left(x^{\prime}\right) \chi_{J M \nu}^{*}(x) .
$$

We note the corresponding contributions

$$
\left[-\epsilon\left(x x^{\prime}\right)-\mathcal{E}_{J \nu} \delta\left(x-x^{\prime}\right)\right] \phi_{J M \nu}(x) \phi_{J M \nu}^{*}\left(x^{\prime}\right) .
$$

Applying Eqs. (4.13) and (4.14) to the interaction term, we find at the stage that the sums over $I M$ and $I^{\prime} M^{\prime}$ have been carried out,

$$
\begin{aligned}
& \frac{1}{2} \int d R d R^{\prime} F\left(x x^{\prime} x^{\prime \prime} x^{\prime \prime \prime}\right) D_{M^{\prime} M^{\prime \prime}}^{(J)}\left(R^{-1} R^{\prime}\right) D_{M^{i v} M^{\prime \prime \prime}}^{\left(J^{\prime} *\right.}\left(R^{-1} R^{\prime}\right) \\
& \times \chi_{J M^{\prime} \nu}\left(R y^{\prime}\right) \chi_{J M^{\prime \prime} \nu}^{*}\left(R^{\prime} y\right) \chi_{J^{\prime} M^{\prime \prime \prime} \nu^{\prime}}\left(R^{\prime} x^{\prime}\right) \chi_{J^{\prime} \nu^{i v} \nu^{\prime}}^{*}(R x) .
\end{aligned}
$$

Introducing the definition

$$
R^{-1} R^{\prime}=\mathcal{R},
$$

and replacing the integral over $R^{\prime}$ by an integral $\mathcal{R}$, we could do the integrals exactly by decomposing the amplitudes $\chi$ into irreducible tensors. We have resisted the temptation to do this, since a full calculation was carried out in Sec. III. It is more illuminating, as well as simpler, to proceed approximately by expanding $\mathcal{R}$ about the unit matrix where ever it appears as the argument of a $\chi$ function. This brings in at each order angular momentum operators acting on single-particle wave-functions and therefore dimensionally is the source of the expansion in $(\langle j\rangle / J)$. For the interaction term the cranking limit will arise from the leading term of this expansion.

With the help of the rotational invariance of the interaction, in the present instance the relation for example $F\left(R x R x^{\prime} R x^{\prime \prime} R x^{\prime \prime \prime}\right)=F\left(x x^{\prime} x^{\prime \prime} x^{\prime \prime \prime}\right)$, and the orthonormality relations of the $D$ functions, we reach the result

$$
\frac{1}{2} \sum_{J M M^{\prime} \nu \nu^{\prime}} \frac{1}{2 J+1} F\left(x x^{\prime} x^{\prime \prime} x^{\prime \prime \prime}\right) \chi_{J M \nu^{\prime}}\left(x^{\prime \prime}\right) \chi_{J M^{\prime} \nu^{\prime}}^{*}\left(x^{\prime \prime \prime}\right) \chi_{J M^{\prime} \nu}\left(x^{\prime}\right) \chi_{J M \nu}^{*}(x),
$$

which is almost the cranking limit. The remaining interaction terms may be written down by inspection, namely

$$
\begin{aligned}
& \sum_{J M M^{\prime} \nu \nu^{\prime}} \frac{1}{2 J+1}\left[G\left(x x^{\prime} x^{\prime \prime} x^{\prime \prime \prime}\right) \chi_{J M \nu^{\prime}}\left(x^{\prime \prime}\right) \phi_{J M^{\prime} \nu^{\prime}}^{*}\left(x^{\prime \prime \prime}\right) \phi_{J M^{\prime} \nu}\left(x^{\prime}\right) \chi_{J M \nu}^{*}(x)\right. \\
& \left.-\frac{1}{2} F\left(x^{\prime \prime \prime} x^{\prime} x^{\prime \prime} x\right) \phi_{J M \nu^{\prime}}\left(x^{\prime \prime}\right) \phi_{J M^{\prime} \nu^{\prime}}^{*}\left(x^{\prime \prime \prime}\right) \phi_{J M^{\prime} \nu}\left(x^{\prime}\right) \phi_{J M \nu}^{*}(x)\right] .
\end{aligned}
$$

It remains for us to calculate the Coriolis coupling terms. Remarking that $\bar{E}^{*}(I K)$ is an eigenvalue of $D_{M K}^{(I)}$,

$$
\bar{E}^{*}(I K) D_{M K}^{(I)}=\bar{E}^{*}\left(\vec{I}_{\mathrm{op}}^{2}-K^{2}\right) D_{M K}^{(I)},
$$


using completeness and integrating by parts, we reach an intermediate stage of the calculation, in the form,

$$
-\int d R\left[\bar{E}^{*}\left(\vec{I}_{\mathrm{op}}^{2}-K^{2}\right) D_{M M^{\prime}}^{(J) *}(R) \chi_{J M^{\prime} \nu}(R x)\right] D_{M M^{\prime}}^{(J)} \chi^{*} J M^{\prime \prime} \nu(R x) .
$$

By distribution of the derivatives, $\vec{I}_{\mathrm{op}} \rightarrow \vec{J}_{\mathrm{op}}+\vec{j}_{\mathrm{op}}$, by noting the relation

$$
\vec{j}_{\mathrm{op}}(R) \chi(R x)=-\vec{j}(x) \chi(R x),
$$

and by expansion in powers of $(j / J)$, Eq. (4.22) becomes to first order (in the intrinsic system)

$$
\begin{aligned}
& -\sum_{J M \nu} \bar{E}^{*}\left[J(J+1)-K^{2}\right]\left|\chi_{J M \nu}(x)\right|^{2} \\
& +\left[\frac{\partial \bar{E}^{*}}{\partial J_{i}} D_{M M^{\prime}}^{(J) *}(R)\right]\left[j_{i}(x) \chi_{J M^{\prime} \nu}(R x)\right] D_{M M^{\prime \prime}}^{(J)}(R) \chi_{J M^{\prime \prime} \nu}^{*}(R x)+\ldots
\end{aligned}
$$

The second term, the Coriolis coupling may be evaluated further by substituting the general relation (3.19) and the matrix elements for intrinsic components,

$$
\begin{aligned}
& J_{-} D_{M M^{\prime}}^{(J) *}=\sqrt{\left(J+M^{\prime}\right)\left(J-M^{\prime}+1\right)} D_{M M^{\prime}-1}^{(J) *}, \\
& J_{+} D_{M M^{\prime}}^{(J) *}=\sqrt{\left(J-M^{\prime}\right)\left(J+M^{\prime}+1\right)} D_{M M^{\prime}+1}^{(J) *}
\end{aligned}
$$

yielding

$$
\begin{aligned}
& \sum_{J M \nu} \bar{f}(J K)\left\{\frac{1}{2} \sqrt{(J-M)(J+M+1)} j_{+} \chi_{J M+1 \nu}(x)\right. \\
& \left.\left.+\frac{1}{2} \sqrt{(J+M)(J-M+1)} j_{-\chi_{J M-1 \nu}}(x)+M j_{3} \chi_{J M \nu}(x)\right\} \chi_{J M \nu}^{*}(x)\right\} .
\end{aligned}
$$

The corresponding calculation for the other Coriolis term yields the sum

$$
\begin{aligned}
& -\sum_{J M \nu} \underline{E}^{*}\left[J(J+1)-K^{2}\right]\left|\phi_{J M \nu}(x)\right|^{2} \\
& +\sum_{J M \nu} \underline{f}(J K)\left\{\frac{1}{2} \sqrt{(J-M)(J+M+1)} j_{+} \phi_{J M+1 \nu}(x)\right. \\
& \left.+\frac{1}{2} \sqrt{(J+M)(J-M+1)} j_{-} \phi_{J M-1 \nu}(x)+M j_{3} \phi_{J M \nu}(x)\right\} \phi_{J M \nu}^{*}(x) .
\end{aligned}
$$

The signs in the Coriolis terms (4.27) and (4.28) appear to be reversed compared to those encountered in Eqs. (3.21) and (3.23), but when due account is taken of Eq. (4.23), there is no inconsistency.

We are finally ready to discuss the cranking limit. The essential observation is that once the expansion to leading order in $(\langle j\rangle / J)$ has been made both in the Coriolis coupling and in the interaction terms, the resulting approximate functional $\mathcal{F}$ presents itself as a single sum over $J$. However, angular momentum is still conserved at this juncture. We lose angular momentum conservation by assuming that consistent with the condition $(\langle j\rangle / J)<<1$ we 
may identify $M$ and $K$, i. e., we may neglect the angular momentum transferred to or from the particle, and write, furthermore, ( $\omega$ defined below)

$$
\begin{aligned}
\chi_{J K \nu}(x) & \rightarrow \sqrt{2 J(\omega)+1} \chi_{\omega \nu}(x), \\
\chi_{J K \pm 1 \nu}(x) & \rightarrow \sqrt{2 J(\omega)+1} C_{\mp} \chi_{\omega \nu}(x),
\end{aligned}
$$

i. e., the amplitudes differing in $K$ from the "central value" by a unit are assumed proportional to the central amplitude (which is defined as the cranking amplitude) up to scale factors $C_{\mp}$ discussed below. Similar definitions hold for the $\phi$ amplitudes. The factor

$\sqrt{2 J+1}$ is inserted for convenience, as will be evident from Eq. (4.33) given below.

These assumptions suggest the following definitions of the components of the angular frequency (overline and underline understood)

$$
\begin{aligned}
\omega_{\mp}(K) & =f(J K) C_{\mp} \sqrt{(J \mp K+1)(J \pm K)}, \\
\omega_{3} & =f(J K) K .
\end{aligned}
$$

The introduction of the factors $C_{\mp}$ may appear gratuitous at first sight, but it is needed, as will become especially evident when we treat the triaxial case, to guarantee that in the cranking limit the theorem that the angular velocity is proportional to the angular momentum is valid [38].

Remembering the definition (3.18) and reinstating Cartesian intrinsic coordinates for the Coriolis coupling terms, we obtain the cranking variational expression

$$
\begin{aligned}
{[\mathcal{F} /(2 J(\omega)+1)]=} & \epsilon\left(x x^{\prime}\right) \chi_{\omega \nu}\left(x^{\prime}\right) \chi_{\omega \nu}^{*}(x)-\epsilon\left(x x^{\prime}\right) \phi_{\omega \nu}(x) \phi_{\omega \nu}^{*}\left(x^{\prime}\right) \\
& +\left(\bar{\omega}_{i} j_{i} \chi_{\omega \nu}(x)\right) \chi_{\omega \nu}^{*}(x)+\left(\underline{\omega}_{i} j_{i} \phi_{\omega \nu}(x)\right) \phi_{\omega \nu}^{*}(x) \\
& +\frac{1}{2} F\left(x x^{\prime} x^{\prime \prime} x^{\prime \prime \prime}\right) \chi_{\omega \nu^{\prime}}\left(x^{\prime \prime}\right) \chi_{\omega \nu^{\prime}}^{*}\left(x^{\prime \prime \prime}\right) \chi_{\omega \nu}\left(x^{\prime}\right) \chi_{\omega \nu}^{*}(x) \\
& +G\left(x x^{\prime} x^{\prime \prime} x^{\prime \prime \prime}\right) \chi_{\omega \nu^{\prime}}\left(x^{\prime \prime}\right) \phi_{\omega \nu^{\prime}}^{*}\left(x^{\prime \prime \prime}\right) \chi_{\omega \nu}\left(x^{\prime}\right) \phi_{\omega \nu}^{*}(x) \\
& -\frac{1}{2} F\left(x^{\prime \prime \prime} x^{\prime} x^{\prime \prime} x\right) \phi_{\omega \nu^{\prime}}\left(x^{\prime \prime}\right) \phi_{\omega \nu^{\prime}}^{*}\left(x^{\prime \prime \prime}\right) \phi_{\omega \nu}\left(x^{\prime}\right) \phi_{\omega \nu}^{*}(x) \\
& -\bar{\varepsilon}_{\omega \nu} \chi_{\omega \nu}(x) \chi_{\omega \nu}^{*}(x)-\underline{\varepsilon}_{\omega \nu} \phi_{\omega \nu}(x) \phi_{\omega \nu}^{*}(x) .
\end{aligned}
$$

The equations of motion that follow are number-conserving, and according to the definitions (4.31) and (4.32) allow solutions with principal axis cranking

\section{TRIAXIAL ROTOR: CORE-PARTICLE COUPLING MODEL AND CRANKING LIMIT}

In this section, we assume that states of interest of neighboring even nuclei can be described phenomenologically by a Hamiltonian

$$
\mathcal{H}_{c}=\frac{1}{2} a_{i} I_{i}^{2}+\frac{1}{4} a_{i j}\left\{I_{i}^{2}, I_{j}^{2}\right\}+\ldots
$$

In the calculations to be described below, we shall retain only the first term of $\mathcal{H}_{c}$. The underlying model arises as follows: We assume that we can identify states of the appropriate 
even nucleus as $\left|I M_{I} n \sigma\right\rangle$, which we read as the $n$th state of angular momentum $I$ belonging to a triaxial intrinsic structure $\sigma$. We also define a rotated intrinsic state

$$
|R \sigma\rangle=U(R)|\hat{0} \sigma\rangle
$$

It is part of the definition of the model that the scalar product

$$
\left\langle R \sigma \mid I M_{I} n \sigma\right\rangle \equiv F_{M_{I} n}^{(I)}(R)
$$

satisfies the eigenvalue equation

$$
\mathcal{H}_{c} F_{M_{I} n}^{(I)}=E^{*}(\operatorname{In}) F_{M_{I} n}^{(I)}
$$

Further useful equations satisfied by or defining the model include

$$
\begin{aligned}
\left|I M_{I} n \sigma\right\rangle & =\left|I M_{I} K \sigma\right\rangle c_{K n}^{(I \sigma)} \\
\delta_{n n^{\prime}} & =\sum_{K} c_{K n}^{(I \sigma) *} c_{K n^{\prime}}^{(I \sigma)} \\
\delta_{K K^{\prime}} & =\sum_{n} c_{K n}^{(I \sigma) *} c_{K^{\prime} n}^{(I \sigma)}, \\
\langle R \sigma \mid I M K \sigma\rangle & =\sqrt{\frac{2 I+1}{8 \pi^{2}}} D_{M K}^{(I)}(R) .
\end{aligned}
$$

We turn to the evaluation of the terms in the variational functional $\mathcal{F}$. We shall follow the methods of both Sec. III and Sec. IV, depending on the aim of a particular fragment of the calculation. Starting from the representation

$$
\begin{aligned}
V_{J M \nu}\left(\alpha I M_{I} n \sigma\right) & =\left\langle J M \nu\left|a_{\alpha}\right| R \sigma\right\rangle F_{M_{I} n}^{(I)}(R) \\
& =\left\langle J M \nu\left|a_{\alpha}\right| R \sigma\right\rangle D_{M_{I} K}^{(I)}(R) c_{K n}^{(I \sigma)},
\end{aligned}
$$

we can derive a formula for the current version of the CFP $V$ that is analogous to Eq. (3.9), namely

$$
\begin{aligned}
V_{J M \nu}\left(\alpha ; I M_{I} n \sigma\right)= & \sum_{K \kappa_{a}} \sqrt{\frac{8 \pi^{2}}{2 j_{a}+1}}(-1)^{J-M} \\
& \times\left(I M_{I} J-M \mid j_{a} m_{a}\right)\left(J K j_{a} \kappa_{a} \mid I K+\kappa_{a}\right) \\
& \times(-1)^{j_{a}+\kappa_{a}} \chi_{J K \nu}\left(j_{a} \kappa_{a} \sigma\right) c_{K+\kappa_{a} n}^{(I \sigma)}, \\
(-1)^{j+m} \chi_{J K \nu}(j m \sigma)= & \left\langle J K \nu\left|a_{j m}\right| \hat{0} \sigma\right\rangle .
\end{aligned}
$$

The corresponding formula for the CFP $U$ is

$$
\begin{aligned}
& U_{J M \nu}\left(\alpha I M_{I} n \sigma\right)=\sum_{K \kappa_{a}} \sqrt{\frac{8 \pi^{2}}{2 j_{a}+1}} \\
& \times(-1)^{J-M+j_{a}-\kappa_{a}+j_{a}+m_{a}}\left(I M_{I} J-M \mid j_{a} m_{a}\right) \\
& \times\left(J K j_{a} \kappa_{a} \mid I K+\kappa_{a}\right) \phi_{J K \nu}\left(j_{a} \kappa_{a} \sigma\right) c_{K+\kappa_{a} n}^{(I \sigma)}, \\
& \phi_{J M \nu}\left(j_{a} \kappa_{a} \sigma\right)=\left\langle J M \nu\left|a_{j_{a}-\kappa_{a}}^{\dagger}\right| \hat{0} \sigma\right\rangle \text {. }
\end{aligned}
$$


With these formulas, we find the contributions of the simplest single-particle terms to take the form, in the shell-model or mode representation,

$$
\begin{array}{r}
\sum_{J K \nu j_{a} \kappa_{a}}\left[\left(\epsilon_{a}-\overline{\mathcal{E}}_{J \nu}\right)\left|\chi_{J K \nu}(a)\right|^{2}\right. \\
-\left(\epsilon_{a}+\underline{\mathcal{E}}_{J \nu}\left|\phi_{J K \nu}(a)\right|^{2}\right] .
\end{array}
$$

We study next the term involving the Lagrange multiplier $\bar{E}^{*}(I n)$. Here it is convenient to carry out the calculation by a method analogous to that utilized beginning with Eq. (4.21). With the help of the defining equation (5.4) and a subsequent integration by parts, we have first of all

$$
\bar{E}^{*}(I n) V_{J M \nu}\left(I M_{I} n \sigma\right)=\int d R\left[\mathcal{H}_{c}\left(\hat{I}_{i}\right)\left\langle J M \nu\left|a_{\alpha}\right| R \sigma\right\rangle\right] F_{M_{I} n}^{(I \sigma)}(R) .
$$

With the help of the completeness relation

$$
\sum_{I M_{I} n} F_{M_{I} n}^{(I \sigma)}(R) F_{I M_{I} n}^{(I \sigma) *}\left(R^{\prime}\right)=\delta\left(R-R^{\prime}\right),
$$

we thus find for the total term

$$
\left.\bar{E}^{*}(\operatorname{In} \sigma)\left|V_{J M \nu}\left(\alpha I M_{I} n \sigma\right)\right|^{2}=\int d R\left[\mathcal{H}_{c}\left(\hat{I}_{i}\right)\left\langle J M \nu\left|a_{\alpha}\right| R \sigma\right\rangle\right] J M \nu\left|a_{\alpha}\right| R \sigma\right\rangle^{*} .
$$

The square bracket may be reexpressed as

$$
\mathcal{H}_{c}\left(\hat{I}_{i}\right)\left\langle J M \nu\left|a_{\alpha}\right| R \sigma\right\rangle=\left[\mathcal{H}_{c}\left(\hat{I}_{i}\right) D_{M K}^{(J) *}(R) D_{m_{a} \kappa_{a}}^{\left(j_{a}\right) *}(R)\right]\left\langle J K \nu\left|a_{j_{a} \kappa_{a}}\right| \hat{0} \sigma\right\rangle .
$$

As far as the application of $\mathcal{H}_{c}$ in $(5.18)$ is concerned, we then write

$$
\begin{aligned}
\mathcal{H}_{c}\left(\hat{I}_{i}\right) & \rightarrow \mathcal{H}_{c}\left(\hat{J}_{i}+\hat{j}_{i}\right) \\
& =\mathcal{H}_{c}\left(\hat{J}_{i}\right)+\frac{\partial \mathcal{H}_{c}}{\partial \hat{J}_{i}} \hat{j}_{i}+\ldots
\end{aligned}
$$

and work only to the order indicated explicitly.

At the same time it is convenient to rewrite

$$
\begin{aligned}
\mathcal{H}_{c}\left(\hat{I}_{i}\right)=\frac{1}{4} b_{1}\left(I_{+} I_{-}+I_{-} I_{+}\right)+\frac{1}{4} b_{2}\left(I_{+}^{2}+I_{-}^{2}\right)+\frac{1}{2} b_{3} I_{3}^{2} \\
a_{1}=b_{1}+b_{2}, \quad a_{2}=b_{1}-b_{2}, \quad a_{3}=b_{3} .
\end{aligned}
$$

It is now straightforward to calculate the contributions arising from the two terms of Eq. (5.19). For the first term we find

$$
\begin{aligned}
& -8 \pi^{2}\left\{\left[\frac{1}{2} b_{1}\left[J(J+1)-K^{2}\right]+\frac{1}{2} b_{3} K^{2}\right]\left|\chi_{J K \nu}(a)\right|^{2}\right. \\
& \frac{1}{4} b_{2} \sqrt{(J-K+2)(J-K+1)(J+K-1)(J+K)} \chi_{J K-2 \nu}(a) \chi_{J K \nu}^{*}(a) \\
& +\frac{1}{4} b_{2} \sqrt{(J+K+2)(J+K+1)(J-K-1)(J-K)} \\
& \left.\times \chi_{J K+2 \nu}(a) \chi_{J K \nu}^{*}(a)\right\}
\end{aligned}
$$


and for the second term,

$$
\begin{aligned}
& -8 \pi^{2}\left[\frac{1}{2} b_{1} \sqrt{(J-K+1)(J+K)\left(j_{a}+\kappa_{a}+1\right)\left(j_{a}-\kappa_{a}\right)} \chi_{J K-1 \nu}\left(j_{a} \kappa_{a}+1 \sigma\right)\right. \\
& +\frac{1}{2} b_{1} \sqrt{(J+K+1)(J-K)\left(j_{a}-\kappa_{a}+1\right)\left(j_{a}+\kappa_{a}\right)} \chi_{J K+1 \nu}\left(j_{a} \kappa_{a}-1 \sigma\right) \\
& +\frac{1}{2} b_{2} \sqrt{(J-K+1)(J+K)\left(j_{a}-\kappa_{a}+1\right)\left(j_{a}+\kappa_{a}\right)} \chi_{J K-1 \nu}\left(j_{a} \kappa_{a}-1 \sigma\right) \\
& +\frac{1}{2} b_{2} \sqrt{(J+K+1)(J-K)\left(j_{a}+\kappa_{a}+1\right)\left(j_{a}-\kappa_{a}\right)} \chi_{J K+1 \nu}\left(j_{a} \kappa_{a}+1 \sigma\right) \\
& \left.+b_{3} K \kappa_{a} \chi_{J K \nu}\left(j_{a} \kappa_{a} \sigma\right)\right] \chi_{J K \nu}^{*}\left(j_{a} \kappa_{a} \sigma\right) .
\end{aligned}
$$

Both of these terms can be identified as familiar structures. By means of this identification we shall have achieved both a simpler form for the particle-rotor formalism and for its limiting case, the cranking formalism. First consider Eq. (5.22). Note that the content of Eqs. (5.4) and (5.5) can be rewritten as

$$
\begin{aligned}
\mathcal{H}_{c} D_{M_{I} K}^{(I)} & =D_{M_{I} K^{\prime}}^{(I)}\left(\mathcal{H}_{c}\right)_{K^{\prime} K}, \\
(\mathcal{H})_{K K^{\prime}} c_{K^{\prime} n}^{(I \sigma)} & =E^{*}(I n) c_{K n}^{(I \sigma)} .
\end{aligned}
$$

This eigenvalue equation was associated with even nuclei and thus with integer values of the angular momentum. By analytic continuation, we can define a corresponding eigenvalue equation for odd nuclei as follows:

$$
\left(\mathcal{H}_{c}\left(\hat{J}_{i}\right)\right)_{K K^{\prime}} c_{K^{\prime} \tau}^{(J)}=E^{*}(J \tau) c_{K \tau}^{(J)},
$$

where $J, K$ are now half-integral. We then see that if we introduce a new set of particle amplitudes $\chi_{J \tau \nu}$ by means of the equation

$$
\chi_{J K \nu}(j \kappa)=c_{K \tau}^{(J)} \chi_{J \tau \nu}(j \kappa),
$$

we can transform Eq. (5.22) into the form

$$
-E^{*}(J \tau)\left|\chi_{J \tau \nu}(a)\right|^{2}
$$

Finally, as we did for the axial case, we can combine energy terms by means of a definition

$$
\varepsilon_{J \nu}=\mathcal{E}_{J \nu}+E^{*}(J \tau)
$$

We turn our attention next to Eq. (5.23). We note first that this expression is an expanded version of

$$
-\left[a_{i} \hat{J}_{i} \hat{j}_{i} \chi_{J K \nu}(a)\right] \chi_{J K \nu}^{*}(a),
$$

where $\hat{J}_{i}$ acts on the value of $K$ and $\hat{j}_{i}$ acts on the value of $\kappa_{a}$. Transforming to the new amplitudes $\chi_{J \tau \nu}$, expression $(5.30)$ becomes

$$
-\left[a_{i} \hat{J}_{i} \hat{j}_{i} \chi_{J \tau \nu}(a)\right] \chi_{J \tau \nu}^{*}(a),
$$


where now

$$
\begin{aligned}
\hat{J}_{i} \chi_{J \tau \nu} & =\chi_{J \tau^{\prime} \nu}\left(J \tau^{\prime}\left|J_{i}\right| J \tau\right), \\
\left(J \tau^{\prime}\left|\hat{J}_{i}\right| J \tau\right) & =c_{K^{\prime} \tau^{\prime}}^{(J)}\left(J K^{\prime}\left|J_{i}\right| J K\right) c_{K \tau}^{(J) *} .
\end{aligned}
$$

For the purpose of taking the cranking limit and comparing the forms derived in Sec. IV, we rewrite the results found so far and the corresponding terms involving $\phi$ amplitudes in coordinate space. For this we require only Eqs. (5.27), the corresponding equations

$$
\begin{aligned}
\chi_{J K \nu}(x) & =\langle J K \nu|\hat{\psi}(x)| \hat{0} \sigma\rangle \\
& =c_{K \tau}^{(J)} \chi_{J \tau \nu}(x),
\end{aligned}
$$

and the similar equations for the terms involving $\phi$. We thus find the contributions

$$
\begin{aligned}
& -\bar{\varepsilon}_{J \nu}\left|\chi_{J \tau \nu}(x)\right|^{2}+\epsilon\left(x x^{\prime}\right) \chi_{J \tau \nu}\left(x^{\prime}\right) \chi_{J \tau \nu}^{*}(x)+\bar{a}_{i}\left[J_{i} j_{i}(x) \chi_{J \tau \nu}(x)\right] \chi_{J \tau \nu}^{*}(x) \\
& -\underline{\varepsilon}_{J \nu}\left|\phi_{J \tau \nu}(x)\right|^{2}+\epsilon\left(x x^{\prime}\right) \phi_{J \tau \nu}\left(x^{\prime}\right) \phi_{J \tau \nu}^{*}(x)+\underline{a}_{i}\left[J_{i} j_{i}(x) \phi_{J \tau \nu}(x)\right] \phi_{J \tau \nu}^{*}(x) .
\end{aligned}
$$

The cranking limit of these terms may now be taken by means of the replacements that generalize Eqs. (4.30) and (4.29),

$$
\chi_{J \tau^{\prime} \nu}(x) \rightarrow \sqrt{2 J+1} C_{\tau}\left(\tau^{\prime}\right) \chi_{\omega \nu}(x),
$$

and $\tau^{\prime}$ refers to $\tau$ or any of the values coupled to $\tau$ by the matrices of $\hat{J}_{i}$, with $C_{\tau}(\tau)=1$. This is the essential blurring of angular momentum conservation that takes us from the conserving particle-rotor approximation to the cranking approximation. It allows us as well to define the components of the angular velocity in generalization of Eq. (4.29).

$$
\begin{aligned}
\bar{\omega}_{i}(\tau) & =\bar{a}_{i} \sum_{\tau^{\prime}} C_{\tau}\left(\tau^{\prime}\right)\left(J \tau^{\prime}\left|J_{i}\right| J \tau\right), \\
\tau^{\prime} & =\tau^{\prime}(\tau)
\end{aligned}
$$

As usual, there are corresponding equations for the amplitudes $\phi$.

We may thus replace Eq. (5.36) by its cranking limit

$$
\begin{aligned}
& (2 J+1)\left[-\bar{\varepsilon}_{\omega \nu}\left|\chi_{\omega \nu}(x)\right|^{2}+\epsilon\left(x x^{\prime}\right) \chi_{\omega \nu}\left(x^{\prime}\right) \chi_{\omega \nu}^{*}(x)+\bar{\omega}_{i} j_{i}(x) \chi_{\omega \nu}(x)\right] \chi_{\omega \nu}^{*}(x) \\
& \left.-\underline{\varepsilon}_{\omega \nu}\left|\phi_{\omega \nu}(x)\right|^{2}+\epsilon\left(x x^{\prime}\right) \phi_{\omega \nu}\left(x^{\prime}\right) \phi_{\omega \nu}^{*}(x)+\underline{\omega}_{i} j_{i}(x) \phi_{\omega \nu}(x)\right] \phi_{\omega \nu}^{*}(x),
\end{aligned}
$$

which is indistinguishable in form from the corresponding terms of Eq. (4.33).

It remains for us to compute the contributions of the interaction terms. We consider first an exact calculation analogous to that carried out in Sec. III, starting from the representations (5.10) and (5.12) for the CFP in the triaxial case. It is straightforward to follow the calculations that begin with Eq. (3.24) and culminate with the result (3.28), as soon as one utilizes the orthonormality relations involving the coefficients $c_{K n}^{(I)}$ at the first step. The final result is 


$$
\begin{aligned}
& \sum=\sum_{I^{\prime \prime} J J^{\prime} K K^{\prime} \nu \nu^{\prime} j_{a} \ldots \kappa_{a} \ldots}\left[\frac{1}{2} F_{a a^{\prime} b^{\prime} b}(L) \chi_{J K-\kappa_{b^{\prime}} \nu}\left(b^{\prime}\right) \chi_{J K^{\prime}-\kappa_{b} \nu}^{*}(b) \chi_{J^{\prime} K^{\prime}-\kappa_{a^{\prime}} \nu^{\prime}}\left(a^{\prime}\right) \chi_{J^{\prime} K-\kappa_{a} \nu^{\prime}}^{*}(a)\right. \\
& -\frac{1}{2} F_{b a^{\prime} b^{\prime} a}(L) \phi_{J K-\kappa_{b^{\prime}} \nu}\left(b^{\prime}\right) \phi_{J K^{\prime}-\kappa_{b} \nu}^{*}(b) \phi_{J^{\prime} K^{\prime}-\kappa_{a^{\prime}} \nu^{\prime}}\left(a^{\prime}\right) \phi_{J^{\prime} K-\kappa_{a} \nu^{\prime}}^{*}(a) \\
& \left.+G_{a a^{\prime} b^{\prime} b} \chi_{J K-\kappa_{b^{\prime} \nu} \nu}\left(b^{\prime}\right) \phi_{J K^{\prime}-\kappa_{b} \nu}^{*}(b) \phi_{J^{\prime} K^{\prime}-\kappa_{a^{\prime}} \nu^{\prime}}\left(a^{\prime}\right) \chi_{J^{\prime} K-\kappa_{a} \nu^{\prime}}^{*}(a)\right] \\
& \times(-1)^{\kappa_{b}+\kappa_{b^{\prime}}-1+j_{a^{\prime}}+j_{b}+L+I^{\prime \prime}} \\
& \times \frac{(2 L+1)}{\left(2 J^{\prime}+1\right)}\left\{\begin{array}{ccc}
j_{a^{\prime}} & j_{a} & L \\
j_{b^{\prime}} & j_{b} & I^{\prime \prime}
\end{array}\right\} \\
& \times\left(j_{a}-\kappa_{a} j_{b^{\prime}} \kappa_{b^{\prime}} \mid I^{\prime \prime} \kappa_{b^{\prime}}-\kappa_{a}\right)\left(j_{a^{\prime}}-\kappa_{a^{\prime}} j_{b} \kappa_{b} \mid I^{\prime \prime} \kappa_{b}-\kappa_{a^{\prime}}\right) \\
& \times\left(I^{\prime \prime} \kappa_{b^{\prime}}-\kappa_{a} J K-\kappa_{b^{\prime}} \mid J^{\prime} K-\kappa_{a}\right)\left(I^{\prime \prime} \kappa_{b}-\kappa_{a^{\prime}} J K^{\prime}-\kappa_{b} \mid J^{\prime} K^{\prime}-\kappa_{a^{\prime}}\right) .
\end{aligned}
$$

Superficially, the change compared to Eq. (3.28) is that instead of a fixed value of $K$, we have a double sum over $K$ and $K^{\prime}$. The same expression holds for a finite number of interacting $K$ bands provided the sums are restricted correspondingly.

Finally, we consider the calculation of the interaction term by the method of Sec. IV, needed to obtain the cranking limit. Here, in place of Eqs. (4.8)-(4.10), we utilize the forms

$$
\begin{aligned}
\left(\begin{array}{c}
V_{J M \nu}\left(x M_{I} n\right) \\
U_{J M \nu}\left(x M_{I} n\right)
\end{array}\right) & =\int d R D_{M M^{\prime}}^{(J) *}(R)\left(\begin{array}{c}
\chi_{J M^{\prime} \nu}(R x, \sigma) \\
\phi_{J M^{\prime} \nu}(R x, \sigma)
\end{array}\right) \sqrt{\frac{2 I+1}{8 \pi^{2}}} F_{M_{I} n}^{(I)}(R), \\
\chi_{J M \nu}(R x, \sigma) & =\langle J M \nu|\hat{\psi}(R x)| \hat{0} \sigma\rangle \\
\phi_{J M \nu}(R x, \sigma) & =\left\langle J M \nu\left|\hat{\psi}^{\dagger}(R x)\right| \hat{0} \sigma\right\rangle .
\end{aligned}
$$

Once the full completeness relation (5.16) is utilized instead of the restricted completeness relation (4.12), the calculation mimics the one carried out in Sec. IV. In terms of the amplitudes $\chi_{J \tau \nu}$ and $\phi_{J \tau \nu}$, the result is

$$
\begin{aligned}
& \sum_{J \tau \tau^{\prime} \nu \nu^{\prime}} \frac{1}{2 J+1}\left\{\frac{1}{2} F\left(x x^{\prime} x^{\prime \prime} x^{\prime \prime \prime}\right) \chi_{J \tau \nu^{\prime}}\left(x^{\prime \prime}\right) \chi_{J \tau^{\prime} \nu^{\prime}}^{*}\left(x^{\prime \prime \prime}\right) \chi_{J \tau^{\prime} \nu}\left(x^{\prime}\right) \chi_{J \tau \nu}^{*}(x)\right. \\
& +G\left(x x^{\prime} x^{\prime \prime} x^{\prime \prime \prime}\right) \chi_{J \tau \nu^{\prime}}\left(x^{\prime \prime}\right) \phi_{J \tau^{\prime} \nu^{\prime}}^{*}\left(x^{\prime \prime \prime}\right) \phi_{J \tau^{\prime} \nu}\left(x^{\prime}\right) \chi_{J \tau \nu}^{*}(x) \\
& \left.-\frac{1}{2} F\left(x^{\prime \prime \prime} x^{\prime} x^{\prime \prime} x\right) \phi_{J \tau \nu^{\prime}}(x) \phi_{J \tau^{\prime} \nu^{\prime}}^{*}\left(x^{\prime}\right) \phi_{J \tau^{\prime} \nu}\left(x^{\prime \prime \prime}\right) \phi_{J \tau \nu}^{*}\left(x^{\prime \prime}\right)\right\} .
\end{aligned}
$$

The cranking limit of this expression is indistinguishable from the corresponding terms of Eq. (4.33) just as was the case for the single-particle terms (5.40). Thus the form of the cranking variational principle for the triaxial is indistinguishable from that for the axial case and need not be written again. It is understood, however, that we are dealing with full three-dimensional cranking, and that the single-particle wave functions have suitably modified symmetry.

\section{SUMMARY AND DISCUSSION}

We have studied the microscopic foundations of the particle-rotor model and of the cranking model for both axial and triaxial nuclei. The microscopic model was chosen in a form in which the interaction is given at the outset as a sum of multipole and pairing forces. 
We carried out the study from the point of view of the Kerman-Klein method based on the equations of motion for single fermion operators, and this choice of interaction has the advantage that the c-number equations of motion for the CFP are formally exact. These equations of motion and an associated variational principle, worked out in Sec. II, form the basis for the remaining considerations.

[In the earliest papers on the KK approach [8, 6], a more general shell-model interaction was used in the derivation of c-number equations. An essential part of the derivation involved the introduction of the physical arguments needed to separate this interaction into multipole and pairing contributions. As a consequence of the limitations of this procedure, the equations of motion found from it are not exact. Nevertheless, the final equations are formally equivalent to those utilized in this paper. The explanation for this concordance is that in the approach in this paper, the "error" involved in the separation has already been built into the starting Hamiltonian, as a further compromise, widely accepted, in the definition of the microscopic theory.]

As the first application, we derived in Sec. III a self-consistent particle-rotor model for axially symmetric nuclei. The derivation was carried out using basic ideas developed in [13], where, starting from a semi-microscopic version of the theory, we derived the standard non-self-consistent version of the particle-rotor model. The present discussion complements the previous one in the sense that it starts from the beginning and carries the reasoning up to the edge of the semi-microscopic form.

We began this work with the prejudice that, as apposed to previous treatments, a natural path to the cranking model involved passing through the particle-rotor model. Though we were ultimately able to confirm this prejudice, the version of the particle-rotor model derived in Sec. III, though a useful one for applications [13], does not appear to be useful in the further transition to cranking. For this purpose we must be able to expand all contributions in powers of $(\langle j\rangle / J)$, the ratio of a characteristic single-particle angular momentum to the collective angular momentum. We have not discovered such an expansion for the interaction forms derived in this first treatment. Therefore, in Sec. IV we start anew, utilizing an approach already described briefly for two-dimensional rotations in an early publication [25]. Rather than pushing through to a formally exact result, we stop the calculation at the leading order of the small parameter, and thus obtain an approximate version of the particle-rotor model that still conserves angular momentum, but is only a step away from the cranking limit. This further step violates angular momentum conservation by the way in which an angular velocity is introduced to replace the collective angular momentum. In Sec. V the considerations of both previous sections are generalized to the triaxial case.

Several special features of our treatment should be highlighted. For the axial case, as soon as the neighboring even nuclei are represented by bands with non-vanishing $K$ values, we have tilted cranking in its simplest form. A fortiori, in the triaxial case we derive the possibility of full three-dimensional cranking. Within our mode of analysis, these statements may be taken to have the status of theorems. Another feature of our derivations of cranking models is that number conservation is maintained.

Nevertheless, in the light of recent developments associated with tilted cranking [17 21], possible limitations on our work have to be addressed. Superficially, our results apply to one quasiparticle spectra of odd nuclei, whereas the current focus of interest is on at least two quasiparticle spectra of even nuclei, and even more on multi-quasiparticle states. In principle, however, these examples are covered by our considerations. Thus the two quasiparticle case is readily derived from the formalism developed in Appendix A. The multiple quasiparticle 
case is covered if one replaces the reference ground states of the even nuclei by suitably chosen band heads of two quasiparticle bands. Details of such calculations are best addressed within the framework of specific applications.

\section{APPENDIX A: FURTHER DEVELOPMENT OF THE THEORY: ITERATIVE SOLUTION SCHEMES}

The theory developed in Sec. II was sufficient for the purposes of the remainder of the body of this work, a study of the strong-coupling limit. However, we cannot resist the temptation to show how this theory can be developed further, and the full architecture used to suggest algorithms for the solution of the non-linear problem thus defined. We point out before proceeding, moreover, that applications of algorithms similar to some of those to be described were carried out in our early work [39,40].

To begin the extended development, it is helpful to introduce a more concise representation of the equations of motion (2.12) and (2.13) for the CFP by defining the vector

$$
\Psi_{i}(\alpha n)=\left(\begin{array}{c}
V_{i}(\alpha n) \\
U_{i}(\alpha n)
\end{array}\right)
$$

The equations of motion can then be written

$$
\mathcal{E}_{i} \Psi_{i}(\alpha n)=\mathcal{H}\left(\alpha n, \beta n^{\prime}\right) \Psi_{i}\left(\beta n^{\prime}\right)
$$

where $\mathcal{H}\left(\alpha n, \beta n^{\prime}\right)$ is the Hermitian matrix

$$
\begin{aligned}
& \mathcal{H}\left(\alpha n, \beta n^{\prime}\right)= \\
& \left(\begin{array}{cc}
\left(\epsilon_{a}^{\prime}-E_{\bar{n}}^{*}\right) \delta_{n n^{\prime}} \delta_{\alpha \beta}+\bar{\Gamma}\left(\alpha n, \beta n^{\prime}\right) & \Delta\left(\alpha n, \beta n^{\prime}\right) \\
\Delta^{*}\left(\beta n^{\prime}, \alpha n\right) & \left(-\epsilon_{a}^{\prime \prime}-E_{\underline{n}}^{*}\right) \delta_{n n^{\prime}} \delta_{\alpha \beta}+\underline{\Gamma}\left(\alpha n, \beta n^{\prime}\right)
\end{array}\right),
\end{aligned}
$$

and the potentials are defined as

$$
\begin{aligned}
\bar{\Gamma}\left(\alpha n, \gamma n^{\prime}\right) & =F_{\alpha \gamma \delta \beta}\left[V_{i}^{*}\left(\beta n^{\prime}\right) V_{i}(\delta n)\right] \\
& =F_{\alpha \gamma \delta \beta} \mathcal{R}_{11}\left(\delta n, \beta n^{\prime}\right), \\
\underline{\Gamma}\left(\alpha n, \gamma n^{\prime}\right) & =F_{\bar{\delta} \bar{\beta} \bar{\alpha} \bar{\gamma}}\left[U_{i}^{*}\left(\beta n^{\prime}\right) U_{i}(\delta n)\right] \\
& =F_{\bar{\delta} \bar{\beta} \bar{\alpha} \bar{\gamma}} \operatorname{tr} \frac{1}{2}\left(1-\tau_{3}\right) \mathcal{R}_{22}\left(\delta n, \beta n^{\prime}\right), \\
\Delta\left(\alpha n, \gamma n^{\prime}\right) & =G_{\alpha \bar{\gamma} \beta \bar{\delta}}\left[U_{i}^{*}\left(\delta n^{\prime}\right) V_{i}(\beta n)\right] \\
& =G_{\alpha \bar{\gamma} \beta \bar{\delta}} \mathcal{R}_{12}\left(\beta n, \delta n^{\prime}\right) .
\end{aligned}
$$

Here we have utilized a generalized density matrix, $\mathcal{R}\left(\alpha n, \beta n^{\prime}\right)$, defined as

$$
\mathcal{R}\left(\alpha n, \beta n^{\prime}\right)=\Psi_{i}(\alpha n) \Psi_{i}^{*}\left(\beta n^{\prime}\right)=\left(\begin{array}{ll}
\mathcal{R}_{11} & \mathcal{R}_{12} \\
\mathcal{R}_{21} & \mathcal{R}_{22}
\end{array}\right),
$$

satisfying the idempotent condition

$$
\mathcal{R}^{2}=\Omega \mathcal{R}
$$


The structure displayed in the preceeding paragraph suggests that if we knew the potentials $\bar{\Gamma}, \underline{\Gamma}, \Delta$, the chemical potential $\lambda$, and the excitation energies $E_{\bar{n}}^{*}, E_{\underline{n}}^{*}$, we could view Eq. (A2) as a linear eigenvalue problem, with given Hamiltonian $\mathcal{H}$, with eigenvalues $\mathcal{E}_{i}$ and with solutions normalized according to Eq. (2.26). In turn, this suggests at least the first elements of an iterative scheme for the solution of Eq. (A2), where given the $\nu$ th approximation, $\mathcal{H}^{(\nu)}$ to $\mathcal{H}$, we define the $(\nu+1)$ st approximation as the solution of the linear eigenvalue equation

$$
\mathcal{E}_{i}^{(\nu+1)} \Psi_{i}^{(\nu+1)}=\mathcal{H}^{(\nu)} \Psi_{i}^{(\nu+1)} .
$$

Thus, with the help of Eq. (A7), we can construct a (tentative, see below) $(\nu+1)$ st approximation, $\mathcal{R}^{(\nu+1)}$, to the density matrix. Consequently, we can calculate a next approximation to the "potentials".

But how do we generate higher approximations to the energies, including $\lambda$, of the neighboring even nuclei also needed for the next approximation to $\mathcal{H}$ ? Actually the formalism provides several alternatives on how to proceed at this point. We describe first a method which adds the minimum number of conditions sufficient to determine the energies. This is to calculate, e. g.,

$$
\begin{aligned}
& E_{\bar{n}}=\langle\bar{n}|H| \bar{n}\rangle \approx E_{\bar{n}}\left(\mathcal{R}^{(\nu+1)}\right)=E_{\bar{n}}^{(\nu+1)}, \\
& E_{\underline{n}}=\langle\underline{n}|H| \underline{n}\rangle \approx E_{\underline{n}}\left(\mathcal{R}^{(\nu+1)}\right)=E_{\underline{n}}^{(\nu+1)},
\end{aligned}
$$

from which we can form the averages and differences needed for the calculation of $\mathcal{H}^{(\nu+1)}$.

Before we can actually proceed to the next iteration, we must take another step first recognized in our early work on the pairing problem [39]. The general theory requires that if we are to interpret the diagonal elements of $H$ as eigenvalues, then the off-diagonal elements of $H$ must vanish. The latter may be calculated at any stage of approximations by the same techniques used for the diagonal elements. In general, we shall find at any intermediate stage of the calculation that these conditions are not fully satisfied. To rectify this deficiency, we must therefore carry out orthogonal transformations in the spaces $|\bar{n}\rangle$ and $|\underline{n}\rangle$ in order to eliminate the off-diagonal elements. These are equivalent to (different) linear transformations of the CFP $V$ and $U$. Such transformations are norm preserving.

We have now (almost) defined a cycle of the present algorithm. To achieve a full measure of self-consistency requires yet an additional link in the chain of reasoning. This is because to this point, the vectors $\Psi_{i}$ cannot be guaranteed to satisfy the normalization conditions (2.28). In general we shall find

$$
\sum_{m_{a} n} \Psi_{i}^{\dagger}(\alpha n) \Psi_{i}(\alpha n)=\Lambda_{a} \Omega_{a}
$$

whereas full self-consistency requires $\Lambda_{a}=1$. We can rectify the deficiency by rescaling the solution

$$
\Psi_{i}^{(\nu)}(\alpha n) \rightarrow \sqrt{\Lambda_{a}} \Psi_{i}^{(\nu)}(\alpha n)
$$

redefining the potentials, e. g.,

$$
\bar{\Gamma}\left(\alpha n, \gamma n^{\prime}\right)=F_{\alpha \gamma \delta \beta} \sqrt{\Lambda_{b} \Lambda_{d}}\left[V_{i}^{*(\nu)}\left(\beta n^{\prime}\right) V_{i}^{(\nu)}(\delta n)\right]
$$


and similarly for other potentials. This requires us to fit in an additional set of iterations until the values $\Lambda_{a}=1$ are achieved.

We next describe an alternative algorithm in which the equations of motion and normalization conditions are used in the same way as in the algorithm just described. However the transition from $\mathcal{H}^{(\nu)}$ to $\mathcal{H}^{(\nu+1)}$ is done differently and requires further development of the formalism. The first step is to combine the equations of motion (A2) with their complex conjugate equations so as to eliminate the eigenvalues $\mathcal{E}_{i}$. We thereby obtain the following equations for the generalized density matrix $\mathcal{R}$,

$$
0=\mathcal{R}\left(\alpha n, \gamma n^{\prime \prime}\right) \mathcal{H}\left(\gamma n^{\prime \prime}, \beta n^{\prime}\right)-\mathcal{H}\left(\alpha n, \gamma n^{\prime \prime}\right) \mathcal{R}\left(\gamma n^{\prime \prime}, \beta n^{\prime}\right)
$$

i. e., we find the vanishing of the commutator, $[\mathcal{R}, \mathcal{H}]=0$.

Before undertaking the exposition of the algorithm, we exhibit an alternative derivation of Eq. (A15) utilizing a variant of the variational principle (2.18), (2.19). Consider the functional

$$
\mathcal{D}=\mathcal{G}-\Theta\left(\alpha n, \beta n^{\prime}\right)\left[\mathcal{R}^{2}\left(\beta n^{\prime}, \alpha n\right)-\Omega \mathcal{R}\left(\beta n^{\prime}, \alpha n\right)\right]
$$

where the "new" constraint with Lagrange multiplier matrix $\Theta$ is for normalization in the density matrix form. Since

$$
\frac{\delta \mathcal{G}}{\delta \mathcal{R}\left(\beta n^{\prime}, \alpha n\right)}=\mathcal{H}\left(\alpha n, \beta n^{\prime}\right),
$$

it follows that the variational condition applied to Eq. (A16) yields the equation

$$
\mathcal{H}-\Theta \mathcal{R}-\mathcal{R} \Theta+\Theta \Omega=0
$$

From this condition, Eq. (A15) is readily derived by forming the appropriate commutator.

However, Eq. (A18) contains additional information that we shall exploit below. Indeed, we shall consider the possibility of constructing an algorithm on the basis of Eq. (A18), but first we describe one that utilizes Eq. (A15) for the density matrix. Recalling the first algorithm described, let us imagine ourselves at the point where we have an approximation to the density matrix $\mathcal{R}$ that has been determined from the iterative procedure associated with the equations of motion (A2), and that there remains the problem of computing the next approximation to the energies of the even neighbors. An alternative to the procedure that starts with Eq. (A10) is to note that the equation of motion in the density matrix form (A15) provides a sufficient set of equations to determine the excitation energies when supplemented by the number conservation conditions

$$
\begin{aligned}
0 & =\sum_{\alpha i}\left|V_{i}(\alpha n)\right|^{2}-\bar{N} \\
& =\sum_{\alpha} \mathcal{R}_{11}(\alpha n, \alpha n)-\bar{N} \\
0 & =\sum_{\alpha i}\left|U_{i}(\alpha n)\right|^{2}-\Omega+\underline{N} \\
& =\sum_{\alpha} \mathcal{R}_{22}(\alpha n, \alpha n)-\Omega+\underline{N}
\end{aligned}
$$


Just as we did previously for the Hamiltonian, we should check that the number operator matrices $\sum_{\alpha} \mathcal{R}_{11}\left(\alpha n, \alpha n^{\prime}\right)$ and $\sum_{\alpha} \mathcal{R}_{22}\left(\alpha n, \alpha n^{\prime}\right)$ are diagonal and, if necessary, diagonalize them so as to improve the convergence of the procedure.

So far we have suggested two possible algorithms which used the equations of motion (A2), attached in each case to a different method for calculating the energies of the even nuclei. We next propose two additional algorithms, also distinguished by one of the two methods of computing the energy in which, however, we replace the equations of motion for the $\Psi$ vectors by a condition related to the density-matrix formulation. Starting from Eq. (A18) we derive the pair

$$
\mathcal{R H}=\mathcal{R} \Theta \mathcal{R}=\mathcal{H} \mathcal{R}
$$

which imply that

$$
\mathcal{H}=\Theta \Omega
$$

Substituting this result into Eq. (A18), we obtain

$$
\mathcal{H}-\frac{1}{\Omega}\{\mathcal{R}, \mathcal{H}\}=0 .
$$

We describe an algorithm based on Eq. (A23) and the normalization condition $\mathcal{R}^{2}=\Omega \mathcal{R}$. Suppose that in a $\nu$ th approximation, we have

$$
\begin{aligned}
\left(\mathcal{R}^{(\nu))}\right)^{2}-\Omega \mathcal{R}^{(\nu)} & =0, \\
\mathcal{H}^{(\nu)}-\frac{1}{\Omega}\left\{\mathcal{R}^{(\nu)}, \mathcal{H}^{(\nu)}\right\} & =\mathcal{Y}^{(\nu)} .
\end{aligned}
$$

As an illustration of the method of steepest descents, we now choose, in order to improve the value of the density matrix

$$
\delta \mathcal{R}^{(\nu)}=-\eta \mathcal{Y}^{(\nu)}
$$

where $\eta$ is an arbitrary small parameter that may be set to unity for $\mathcal{Y}^{(\nu)}$ sufficiently small. We can check that this choice preserves the norm to first order, since it satisfies the required condition

$$
\left\{\mathcal{R}^{(\nu)}, \delta \mathcal{R}^{(\nu)}\right\}-\Omega \delta \mathcal{R}^{(\nu)}=0,
$$

that follows from Eq. (A24). To see this, note that Eq. (A27) implies that

$$
\mathcal{R}^{(\nu)} \delta \mathcal{R}^{(\nu)} \mathcal{R}^{(\nu)}=0
$$

which besides the trivial solution, is satisfied by Eq. (A26), as follows from Eq. (A25).

This as far as we can go without attacking specific models. 


\section{REFERENCES}

[1] A. Bohr and B. R. Mottelson, Nuclear Structure, (Benjamin, New York, 1975).

[2] P. Ring and P. Schuck, The Nuclear Many Body Problem (Springer, Berlin, 1980).

[3] M. S. Sarker, Phys. Rev. C 60, 064309 (1999).

[4] J. Meyer-ter-Vehn, Nucl. Phys. A 249, 111 and 141 (1975).

[5] S. E. Larsson, G. Leander, and I. Ragnarsson, Nucl, Phys. A 307, 189 (1978).

[6] I. Ragnarsson and P. B. Semmes, Hyp. Int. 43, 425 (1988).

[7] G. Do Dang et al, Nucl. Phys. A 114, 481 (1968).

[8] A. Klein and A. Kerman, Phys. Rev. 132, 1326 (1963).

[9] L. Celenza, A. Klein, and A. K. Kerman, Phys. Rev. 140, B245 (1965).

[10] A. Klein, Adv. in Part. and Nucl. Phys 10, 39 (1983).

[11] A. Klein, Phys. Rev. C 30, 1960 (1984).

[12] A. Klein and N. R. Walet, in International Workshop Nuclear Structure Models, edited by R. Bengtsson, J. Draayer, and W. Nazarewicz (World Scientific, Singapore, 1992), p. 229.

[13] P. Protopapas and A. Klein, Phys. Rev. C 55, 699 (1997).

[14] D. R. Inglis, Phys. Rev. 96, 1059 (1954); 103, 1786 (1956).

[15] D. J. Thouless and J. G. Valatin, Nucl. Phys. 31, 211 (1962).

[16] R. Bengtsson and S. Frauendorf, Nucl. Phys. A 314, 27 (1979).

[17] S. Frauendorf, submitted to Rev. Mod. Phys.

[18] S. Fauendorf, Nucl. Phys. A 557, 259c (1993).

[19] S. Frauendorf and J. Meng, Z. Phys. A 356, 263 (1996).

[20] S. Frauendorf and J. Meng, Nucl. Phys. A 617, 131 (1997).

[21] S. Frauendorf, Z. Phys. A 358, 163 (1997).

[22] P. Ring, R. Beck, and H. J. Mang, Z. Phys. bf 231,10 (1970).

[23] R. Beck, H. J. Mang, and P. Ring, Z. Phys. 231, 26 (1970).

[24] A. Kamlah, Z. Phys. bf 216, 52 (1968).

[25] R. Dreizler and A. Klein, Phys. Rev. Lett. 15, 893 (1965).

[26] A. Klein and M. G. Vassanji, Nucl. Phys. A 317, 116 (1979).

[27] A. Klein and M. G. Vassanji, Phys. Rev. Lett. 42, 436 (1978).

[28] G. Do Dang et al, Nucl. Phys. A 114, 501 (1968).

[29] D. Janssen, Sov. J. Nucl. Phys. 28, 479 (1978).

[30] M. Born and P. Jordan, Z. Phys. 34, 858 (1925).

[31] W. R. Greenberg, A. Klein, I. Zlatev, and C. T. Li, Phys. Rev. A 54, 1820 (1996).

[32] R. Courant and D. Hilbert, Methods of Mathematical Physics, (Interscience, New York, 1965), Vol. 1, p. 459.

[33] A. K. Theophilou, J. Phys. C 12, 5419 (1979).

[34] E. K. U. Gross, L. N. Oliveira, and W. Kohn, Phys. Rev. A 37, 2805 (1988); 37, 2809 (1988); 37, 2821 (1988).

[35] S. L. Adler, Nucl. Phys. B 415, 195 (1994).

[36] D. M. Brink et al, J. Phys. G: Nucl. Phys. 13, 629 (1987).

[37] E. R. Edmonds, Angular Momentum in Quantum Mechanics (Princeton University Press, Princeton, NJ, 1960).

[38] A. K. Kerman and N. Onishi, Nucl. Phys. A 361, 179 (1981).

[39] G. Do Dang et al, Phys. Rev. 172, 1022 (1968). 
[40] G. J. Dreiss et al, Phys. Rev. C 3, 2412 (1971). 